\title{
TESTING INDEPENDENCE WITH HIGH-DIMENSIONAL CORRELATED SAMPLES
}

\begin{abstract}
BY XI CHEN AND WEIDONG LIU ${ }^{1}$
New York University and Shanghai Jiao Tong University

Testing independence among a number of (ultra) high-dimensional random samples is a fundamental and challenging problem. By arranging $n$ identically distributed $p$-dimensional random vectors into a $p \times n$ data matrix, we investigate the problem of testing independence among columns under the matrix-variate normal modeling of data. We propose a computationally simple and tuning-free test statistic, characterize its limiting null distribution, analyze the statistical power and prove its minimax optimality. As an important by-product of the test statistic, a ratio-consistent estimator for the quadratic functional of a covariance matrix from correlated samples is developed. We further study the effect of correlation among samples to an important high-dimensional inference problem-large-scale multiple testing of Pearson's correlation coefficients. Indeed, blindly using classical inference results based on the assumed independence of samples will lead to many false discoveries, which suggests the need for conducting independence testing before applying existing methods. To address the challenge arising from correlation among samples, we propose a "sandwich estimator" of Pearson's correlation coefficient by de-correlating the samples. Based on this approach, the resulting multiple testing procedure asymptotically controls the overall false discovery rate at the nominal level while maintaining good statistical power. Both simulated and real data experiments are carried out to demonstrate the advantages of the proposed methods.
\end{abstract}

1. Introduction. The independence among samples is a fundamental assumption in most statistical modeling upon which numerous estimation and inference methods and theories have been developed. Indeed, from classical statistical inference (e.g., Student's $t$-test) to popular topics in modern statistics (e.g., high-dimensional problems, such as regression, matrix estimation and inference), this assumption of independence occurs widely. Consider $n$ samples $\boldsymbol{X}_{1}, \ldots, \boldsymbol{X}_{n}$, where each sample is a $p$-dimensional vector from the same population distribution with mean $\boldsymbol{\mu} \in \mathbb{R}^{p}$ and covariance $\boldsymbol{\Sigma}=\left(\sigma_{i j}\right)_{p \times p}$. It is often convenient to pool

Received November 2015; revised March 2017.

${ }^{1}$ Supported by NSFC Grant No. 11431006, the Program for Professor of Special Appointment (Eastern Scholar) at Shanghai Institutions of Higher Learning, Shanghai Shuguang Program, Youth Talent Support Program, 973 Program (2015CB856004) and a grant from Australian Research Council.

MSC2010 subject classifications. Primary 62F05; secondary 62H10.

Key words and phrases. Independence test, multiple testing of correlations, false discovery rate, matrix-variate normal, quadratic functional estimation, high-dimensional sample correlation matrix. 
$n$ samples together to form a $p \times n$ data matrix $\mathbf{X}=\left(\boldsymbol{X}_{1}, \ldots, \boldsymbol{X}_{n}\right)$. More specifically, for example, in microarray data, $\mathbf{X}$ is an expression level matrix for $p$ genes measured on $n$ subjects. Such data are usually high-dimensional; thus, we mainly consider the setting where $p$ is much larger than $n$. Most existing works in highdimensional literature make the independence assumption among columns of $\mathbf{X}$, serving as the starting point of methodology development and technical analysis. However, recent studies have shown that there are correlation structures among subjects in various microarray datasets [see, e.g., Allen and Tibshirani (2012), Efron (2009), Kim et al. (2012), Teng and Huang (2009)], demonstrating the potential risk of making the seemingly natural assumption of independence. Therefore, given a data matrix $\mathbf{X}$, it is important to first test whether the samples are indeed independent before applying any method that assumes independence.

A data matrix $\mathbf{X}=\left(\boldsymbol{X}_{1}, \ldots, \boldsymbol{X}_{n}\right)$ is known as transposable data when both rows and columns are potentially correlated [Lazzeroni and Owen (2002), Allen and Tibshirani (2012)]. For a transposable data matrix $\mathbf{X}$, it is commonly assumed that $\mathbf{X}$ follows a matrix-variate normal distribution, which has been widely applied to model microarray data [see, e.g., Allen and Tibshirani (2012), Efron (2009), Kim et al. (2012), Muralidharan (2010), Teng and Huang (2009), Yin and Li (2012), Zhou (2014)]. The matrix-variate normal distribution is a natural generalization of familiar vector-variate normal distribution [Dawid (1981)]. In particular, let $\operatorname{vec}(\mathbf{X}) \in \mathbb{R}^{n p \times 1}$ be the vectorization of matrix $\mathbf{X}$ obtained by stacking the columns of $\mathbf{X}$ on top of each other. We say $\mathbf{X} \in \mathbb{R}^{p \times n}$ follows a matrix-variate normal distribution with the mean matrix $\mathbf{M} \in \mathbb{R}^{p \times n}$ and covariance matrix $\boldsymbol{\Sigma} \otimes \boldsymbol{\Psi} \in \mathbb{R}^{n p \times n p}$ [denoted by $\mathbf{X} \sim N(\mathbf{M}, \boldsymbol{\Sigma} \otimes \boldsymbol{\Psi})$ ] if and only if $\operatorname{vec}\left(\mathbf{X}^{\prime}\right) \sim N\left(\operatorname{vec}\left(\mathbf{M}^{\prime}\right), \boldsymbol{\Sigma} \otimes \boldsymbol{\Psi}\right)$. Here, $\mathbf{X}^{\prime}$ denotes the transpose of $\mathbf{X}, \otimes$ is the Kronecker product and $\boldsymbol{\Psi}=\left(\psi_{i j}\right)_{n \times n} \in \mathbb{R}^{n \times n}$ is the covariance matrix of row vectors of $\mathbf{X}$. Given a matrix-variate normal $\mathbf{X} \sim N(\mathbf{M}, \boldsymbol{\Sigma} \otimes \boldsymbol{\Psi})$, each column $\boldsymbol{X}_{i} \sim N\left(\boldsymbol{M}_{i}, \psi_{i i} \boldsymbol{\Sigma}\right)$ for $1 \leq i \leq n$, where $\boldsymbol{M}_{i}$ is the $i$ th column of the mean matrix M. Recall our problem setup: each $\boldsymbol{X}_{i}$ follows the same population distribution with mean vector $\boldsymbol{\mu}$ and covariance $\boldsymbol{\Sigma}$. Thus, we have $\mathbf{M}=\boldsymbol{\mu} \mathbf{1}^{\prime}$ where $\mathbf{1}$ is the $n$-dimensional all one column vector and $\psi_{i i}=1$ for $1 \leq i \leq n$. Under the matrix-variate normal modeling of the data, the independence testing problem is equivalent to the global test of whether $\boldsymbol{\Psi}$ is a diagonal matrix, that is,

$$
H_{0}: \quad \psi_{i j}=0 \quad \text { for all } 1 \leq i<j \leq n .
$$

The testing problem in (1) is closely related to the following correlation test problem:

$$
H_{0}: \quad \rho_{i j}=0 \quad \text { for all } 1 \leq i<j \leq p,
$$

where $\rho_{i j}=\sigma_{i j} / \sqrt{\sigma_{i i} \sigma_{j j}}$ is the Pearson's correlation coefficient. The testing problem in (2) is a classical problem in multivariate analysis [Anderson (2003), Nagao (1973)]. It has also been extensively studied in the past decade under the highdimensional setting [e.g., Johnstone (2001), Ledoit and Wolf (2002), Jiang (2004), 
Schott (2005), Liu, Lin and Shao (2008), Bai et al. (2009), Cai and Jiang (2011), Jiang and Yang (2013), Han and Liu (2017)]. However, the reported results are based on the assumption that samples are independent. In fact, our problem in (1) is equivalent to the testing problem (2) with correlated samples. To see this, note that when treating each row of $\mathbf{X}$ as an individual sample, the role of $\boldsymbol{\Psi}$ and $\boldsymbol{\Sigma}$ interchanges since $\mathbf{X}^{\prime} \sim N\left(\mathbf{1} \boldsymbol{\mu}^{\prime}, \boldsymbol{\Psi} \otimes \boldsymbol{\Sigma}\right)$, that is, the matrix $\boldsymbol{\Sigma}$ models the correlations among row samples while $\boldsymbol{\Psi}$ becomes the population covariance matrix. For many types of data (e.g., genetic data, financial data), there exists a complicated correlation structure among $p$ variables. Thus, $\boldsymbol{\Sigma}$ will not be a diagonal matrix and row vectors are not independent. Our problem in (1) essentially tests the correlation among row vectors when samples are correlated. The correlation among samples makes our problem more challenging; and the aforementioned methods for testing (2), which are based on the assumption of sample independence, cannot be applied to our problem.

The classical methods for testing independence among samples commonly assume $p$ is fixed and are usually designed only for time series data. It is also known as serial independence test; see Hong (1998) and the references therein. In such a framework, the methods require that the samples under alternatives come from some time series. These samples satisfy an ordering structure such that the dependence between two samples decays as the distance of their indices increases. In our setting, there is no structural assumption among samples. Without any structural assumption, we will show in Theorem 2.7 that any test will not have the power tending to 1 uniformly over a large class of alternatives when the dimension $p$ is small [e.g., fixed constant or $p=o(\log n)$ ]. On the other hand, for $p \geq c \log n$ but is small compared to $n$, the independence test is relatively easy. In fact, if $\boldsymbol{\Sigma}$ is known, the data matrix can be transformed as $\boldsymbol{\Sigma}^{-1 / 2} \mathbf{X} \sim N\left(\boldsymbol{\Sigma}^{-1 / 2} \boldsymbol{\mu} \mathbf{1}^{\prime}, \mathbf{I}_{p \times p} \otimes \boldsymbol{\Psi}\right)$; and thus the independence test can be directly carried out using existing approaches [e.g., Jiang (2004), Liu, Lin and Shao (2008)]. One can apply such an approach with a plug-in estimator $\widehat{\Sigma^{-1}}$. However, as we will explain later in Section 5, when $p \geq c n$, even the optimal convergence rate of the estimator $\widehat{\Sigma^{-1}}$ is not fast enough to solve this problem. In fact, although we have more information (i.e., row samples) as $p$ becomes larger, the number of unknown parameters in $\boldsymbol{\Sigma}$ increases accordingly, which makes the problem challenging. Therefore, the high-dimensional setting is the most interesting case, and will be the main focus of the paper.

Although the testing of independence among high-dimensional samples is an important and fundamental problem, few existing works have done so. Based on matrix-variate normal modeling of the data, some inference approaches were proposed by Efron (2009) and Muralidharan (2010). However, these works do not explore the limiting null distributions as well as the validity and power of the test. Pan, Gao and Yang (2014) proposed a statistic for this problem based on random matrix theory. However, it requires the condition that $p$ is proportionally as large as $n$ (i.e., $0<\lim _{n \rightarrow \infty} \frac{p}{n}<\infty$ ), and thus cannot be applied to cases where $p=n^{r}$ 
with $r>1$ or, as in the ultra high-dimensional setting, where $p=\exp \left(n^{\gamma}\right)$ for some $0<\gamma<1$; both scenarios are common in genetic applications. Further, the method in Pan, Gao and Yang (2014) requires splitting $n$ samples into two parts and differences in splitting could lead to different test results. In this paper, we consider the (ultra) high-dimensional setup and propose a minimax optimal test procedure in terms of the statistical power for the testing problem in (1). We show that the distribution of the proposed max-type test statistic converges to a type I extreme value distribution under the null (Theorem 2.4). Therefore, the proposed test has the pre-specified significance level asymptotically. We also investigate the statistical power. Roughly speaking, we show that under some very mild conditions on off-diagonal elements of $\boldsymbol{\Psi}$, the power will converge to 1 . Further, we prove that the proposed test is minimax rate-optimal over a large class of $\boldsymbol{\Psi}$ (Theorems 2.5 and 2.6).

Our construction of the test statistic combines a bias correction and a variance correlation based on the sample covariance matrix $\left(\hat{\psi}_{i j}\right)_{n \times n}$, where we treat each row of $\mathbf{X}$ as a sample. The bias correction technique allows us to handle the ultra high-dimensional case. Moreover, the variance correlation technique deals with the correlation structure among "row samples" of $\mathbf{X}$, which is specified by $\boldsymbol{\Sigma}$. To characterize the strength of correlation among row samples, we identify a key quantity $A_{p}=\frac{p\|\boldsymbol{\Sigma}\|_{\mathrm{F}}^{2}}{(\operatorname{tr}(\boldsymbol{\Sigma}))^{2}}$, which comes from the asymptotic variance of our bias-corrected statistic. Here, $\|\cdot\|_{F}$ denotes the Frobenius norm and $\operatorname{tr}(\boldsymbol{\Sigma})$ is the trace of $\boldsymbol{\Sigma}$. To simultaneously control the type I error under null and maintain the minimax rateoptimal statistical power, we need a ratio consistent estimator of $A_{p}$ regardless of the correlation among samples. Therefore, the remaining task essentially reduces to the problem of estimating $\|\Sigma\|_{\mathrm{F}}^{2}$ from correlated samples.

It is noteworthy that estimating $\|\boldsymbol{\Sigma}\|_{\mathrm{F}}^{2}$ itself is an important problem, which is known as quadratic functional estimation of $\boldsymbol{\Sigma}$ [see, e.g., Bai and Saranadasa (1996), Chen and Qin (2010), Fan, Rigollet and Wang (2015)]. Most existing works are based on the assumption that samples are independent and identically distributed (i.i.d.), and thus, cannot be directly applied to our problem. Motivated by the thresholding estimator in Fan, Rigollet and Wang (2015), we propose a plugin estimator for $\|\Sigma\|_{\mathrm{F}}^{2}$ based on a thresholded sample covariance matrix but we relax the independence assumption among samples. Further, we propose a definite threshold level, which is adaptive to the amount of correlations among samples and guarantees the consistency of the resulting estimator. Our simulation results demonstrate the superior performance of the proposed estimator of $\|\boldsymbol{\Sigma}\|_{\mathrm{F}}^{2}$ over the existing approaches, which leads to a significant improvement in statistical power.

In summary, we propose a simple max-type test statistic to conduct the global test of independence among high-dimensional random samples in (1). Our approach has the following advantages:

1. Our construction is direct and computationally attractive, which only requires the row sample covariance matrix $\left(\hat{\psi}_{i j}\right)_{n \times n}$ and a threshold estimator of

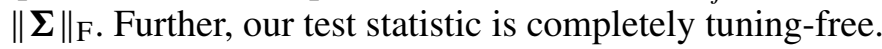


2. The limiting null distribution is characterized, and thus the type I error is controlled asymptotically. Further, our test procedure is minimax rate-optimal over a sufficiently large class of $\boldsymbol{\Psi}$, which is enough for most practical purposes.

3. As an important by-product, we provide a ratio-consistent estimator for estimating quadratic functional of covariance matrix from correlated samples.

We would like to note that we only focus on the matrix-variate normal distribution, which is a common assumption for studying a transposable data matrix and widely used for modeling correlated microarray data. It is of interest to investigate the independence test for more general distributions, for example, a matrix elliptical distribution [Dawid (1977), Fang and Zhang (1990)] or $\mathbf{X}=\boldsymbol{\Sigma}^{1 / 2} \mathbf{Z} \Psi^{1 / 2}$, where entries of $\mathbf{Z}=\left(Z_{i j}\right)_{p \times n}$ are i.i.d. random variables with unit variance. We leave the extension to such distributions of data matrices for future work.

After we conduct the independence test, if the samples are indeed correlated, many classical inference approaches cannot be directly applied. We use the multiple testing problem of Pearson's correlation coefficients to illustrate the effect of the correlation among samples, demonstrate the reason why the classical approach will fail when samples are correlated and further develop a new method to decorrelate the samples. In particular, we consider the following large-scale multiple testing problem, for $1 \leq i<j \leq p$,

$$
H_{0 i j}: \quad \rho_{i j}=0 \quad \text { versus } H_{1 i j}: \quad \rho_{i j} \neq 0 .
$$

Problem (3) is a natural extension of the global test of independence in (2). In fact, the hypothesis that $\boldsymbol{\Sigma}$ is a diagonal matrix is a strong null hypothesis, which will be rejected in most real data applications (e.g., microarray data, stock data). In contrast, the goal of the multiple testing problem (3) is to identity the pairs of correlated variables, and thus find many applications in real data analysis, for example, gene co-expression network analysis [Carter et al. (2004), Hirai et al. (2007), Lee, Hsu and Sajdak (2004), Zhu et al. (2005)], and brain connectivity analysis [Shaw et al. (2006)]. The goal of the testing problem in (3) is consistent with the goal of support recovery of a sparse $\boldsymbol{\Sigma}$. The latter problem has been extensively studied in recent years [e.g., see Rothman, Levina and Zhu (2009), Lam and Fan (2009), Cai and Liu (2011), Bien and Tibshirani (2011)]. These works establish consistency results of support recovery from independent samples under certain conditions, for example, all the absolute values of nonzero $\rho_{i j}$ are lower bounded by $C \sqrt{\frac{\log p}{n}}$, which might be hard to hold in practice. Instead of trying to achieve the perfect support recovery, the multiple testing problem (3) has a more refined control of the type I error rate in support recovery under weaker assumptions. In particular, it usually aims to control the false discovery rate (FDR), which is a useful measure for evaluating the performance of support recovery. We also note that Cai and Liu (2016) recently studied problem (3) in a high-dimensional setting but it still requires the independence assumption. 
For correlated samples from a matrix-variate normal distribution, we first establish the following result on the limiting distribution of the sample correlation coefficient $\hat{\rho}_{i j}$ (see Proposition 3.1):

$$
\frac{\sqrt{n}\left(\hat{\rho}_{i j}-\rho_{i j}\right)}{\sqrt{B_{n}}\left(1-\rho_{i j}^{2}\right)} \Rightarrow N(0,1),
$$

where $B_{n}=\frac{\|\Psi\|_{\mathrm{F}}^{2}}{n}$, which quantifies the strength of the correlation among samples. Equation (4) subsumes as a special case the classical results on the limiting distribution of $\hat{\rho}_{i j}$ when samples are i.i.d. [ $B_{n}=1$ in (4)] [see Theorem 4.2.4 in Anderson (2003)]. When the correlation is strong to a certain extent such that $B_{n}>1+c$ for some constant $c>0$, directly using sample correlation coefficient $\sqrt{n} \hat{\rho}_{i j}$ or Fisher's $z$ statistic will lead to many false positives; this is verified by our simulations in Section 4.2. In fact, even if $B_{n}$ is known and one uses the correct limiting null distribution $N\left(0, \frac{B_{n}}{n}\right)$ of $\hat{\rho}_{i j}$, the variance of $\hat{\rho}_{i j}-\rho_{i j}$ becomes larger as $B_{n}$ increases, which leads to a lower power of the test.

To overcome the side effect of correlation among samples, we propose a "sandwich estimator" of $\rho_{i j}$ by de-correlating the samples, which has the limiting distribution $N\left(\rho_{i j}, \frac{1}{n}\left(1-\rho_{i j}^{2}\right)^{2}\right)$. The corresponding asymptotical variance does not depend on $B_{n}$ and is smaller than that of the naïve estimator $\hat{\rho}_{i j}$. Therefore, the proposed "sandwich estimator" has an improved statistical power especially when the correlation among samples is strong. Based on the proposed "sandwich estimator" of $\rho_{i j}$, the standard multiple testing procedure [Benjamini and Hochberg (1995)] is proven to asymptotically control the FDR at the nominal level (see Theorem 3.2).

Finally, we introduce some necessary notation. For a positive integer $p,[p]:=$ $\{1, \ldots, p\}$. For a square matrix $\mathbf{A}$, let $\operatorname{tr}(\mathbf{A})$ denote the trace of $\mathbf{A}, \lambda_{\max }(\mathbf{A})$ the maximum eigenvalue of $\mathbf{A}$ and $\lambda_{\min }(\mathbf{A})$ the minimum eigenvalue of $\mathbf{A}$. Let $I\{B\}$ be the indicator function that takes value one when the event $B$ is true and zero otherwise. For a given set $\mathcal{H}$, let $\operatorname{Card}(\mathcal{H})$ be the cardinality of $\mathcal{H}$. For any two real numbers $a$ and $b$, let $a \vee b=\max (a, b)$ and $a \wedge b=\min (a, b)$. We use $\overline{\lim }$ and $\underline{\lim }$ to denote limit superior and limit inferior, respectively. Throughout the paper, we use $\mathbf{I}_{p \times p}$ to denote the $p \times p$ identity matrix, and use $C, c, c_{1}$, etc. to denote constants for which values might change from place to place and do not depend on $n$ and $p$.

The rest of the paper is organized as follows. In Section 2, we study the global test in (1). The test statistic is proposed in Section 2.1. In Section 2.2, we provide the ratio consistent estimator of $A_{p}$ and $\|\Sigma\|_{\mathrm{F}}$ from correlated samples. The estimation error is characterized in Theorem 2.1. We further provide the limiting null distribution of the test statistic and the power analysis (Theorems 2.4-2.7). Section 3 studies the multiple testing of correlations in (3) from correlated samples. Experimental results are given in Section 4 followed by discussion in Section 5. Due to space limitations, the proofs of our results as well as some addi- 
tional experimental results are provided in the Supplementary Material [Chen and Liu (2018)].

2. Sample independence test. We study the global testing problem of sample independence in (1) given the $p \times n$ data matrix $\mathbf{X}=\left(\boldsymbol{X}_{1}, \ldots, \boldsymbol{X}_{n}\right) \sim$ $N\left(\boldsymbol{\mu} \mathbf{1}^{\prime}, \boldsymbol{\Sigma} \otimes \boldsymbol{\Psi}\right)$.

2.1. Construction of the test statistic. Recall that $X_{i}=\left(X_{i 1}, \ldots, X_{i p}\right)^{\prime}$ denotes the $i$ th sample for $1 \leq i \leq n$ and let $\bar{X}=\frac{1}{n} \sum_{i=1}^{n} \boldsymbol{X}_{i}=:\left(\bar{X}_{1}, \ldots, \bar{X}_{p}\right)^{\prime}$. Define

$$
\hat{\psi}_{i j}=\frac{1}{p} \sum_{k=1}^{p}\left(X_{i k}-\bar{X}_{k}\right)\left(X_{j k}-\bar{X}_{k}\right), \quad 1 \leq i, j \leq n .
$$

In fact, from the proof, the statistic $\left(\hat{\psi}_{i j}\right)_{n \times n}$ is the sample covariance coefficient corresponding to $\frac{\operatorname{tr}(\boldsymbol{\Sigma})}{p} \psi_{i j}$. Further, under the null $H_{0}$, we can show that

$$
\hat{\psi}_{i j}=\frac{1}{p} \sum_{k=1}^{p}\left(X_{i k}-\mu_{k}\right)\left(X_{j k}-\mu_{k}\right)-\frac{1}{n p} \sum_{k=1}^{p} \sigma_{k k}+O_{\mathbb{P}}\left(\frac{1}{\sqrt{n p}}\right) .
$$

The first term $\frac{1}{p} \sum_{k=1}^{p}\left(X_{i k}-\mu_{k}\right)\left(X_{j k}-\mu_{k}\right)$ has mean $\frac{\operatorname{tr}(\boldsymbol{\Sigma})}{p} \psi_{i j}$ and variance $\frac{\|\boldsymbol{\Sigma}\|_{\mathrm{F}}^{2}}{p^{2}}\left(\psi_{i i} \psi_{j j}+\psi_{i j}^{2}\right)$. The bias term $\frac{1}{n p} \sum_{k=1}^{p} \sigma_{k k}$ comes from the centralization statistics $\left\{\bar{X}_{k}\right\}_{k=1}^{p}$ in (5). When $p=o\left(n^{2}\right)$, we have $\frac{1}{n p} \sum_{k=1}^{p} \sigma_{k k}=o(1 / \sqrt{p})$ and $\sqrt{p} \hat{\psi}_{i j}$ can be shown to converge to a normal distribution. However, as we are interested in the ultra high-dimensional case where $p$ can be as large as $\exp \left(o\left(n^{\gamma}\right)\right)$ for some $0<\gamma<1$, when $p$ becomes larger such that $n^{2}=o(p), \sqrt{p} \hat{\psi}_{i j} \rightarrow-\infty$ in probability under the null. To enable the applicability of our test statistic in the ultra high-dimensional setting, we first propose the following bias corrected quantity:

$$
T_{i j}:=\hat{\psi}_{i j}+\frac{1}{n p} \sum_{k=1}^{p} \hat{\sigma}_{k k},
$$

where $\hat{\sigma}_{k k}=\frac{1}{n-1} \sum_{j=1}^{n}\left(X_{j k}-\bar{X}_{k}\right)^{2}$ is the sample variance corresponding to $\sigma_{k k}$. Since the first term in (6) has variance $\frac{\|\Sigma\|_{\mathrm{F}}^{2}}{p^{2}}\left(\psi_{i i} \psi_{j j}+\psi_{i j}^{2}\right)$, the asymptotic variance of $T_{i j}$ is $\left(\frac{\operatorname{tr}(\boldsymbol{\Sigma})}{p}\right)^{2} \frac{A_{p}}{p}$, where

$$
A_{p}=\frac{p\|\boldsymbol{\Sigma}\|_{\mathrm{F}}^{2}}{(\operatorname{tr}(\boldsymbol{\Sigma}))^{2}}
$$

quantifies the strength of correlations among row vectors of $\mathbf{X}$. 
Given $A_{p}$ in (8), we will show that under the null as $(n, p) \rightarrow \infty$,

$$
\begin{gathered}
\mathbb{P}\left(\frac{p}{A_{p}} \max _{1 \leq i<j \leq n} \frac{T_{i j}^{2}}{\hat{\psi}_{i i} \hat{\psi}_{j j}}-4 \log n+\log \log n \leq t\right) \\
\rightarrow \exp \left(-\frac{1}{\sqrt{8 \pi}} \exp \left(-\frac{t}{2}\right)\right)
\end{gathered}
$$

for $t \in \mathbb{R}$, where the term $A_{p}$ plays the role of variance correction for $T_{i j}$. The remaining task is to develop a ratio consistent estimator $\hat{A}_{p}$ for $A_{p}$. In addition, to maintain the statistical power, the estimator $\hat{A}_{p}$ should also be consistent for correlated samples. In Section 2.2, we will develop such an estimator for $A_{p}$. Given the estimator $\hat{A}_{p}$ [see (14)], we propose the following test statistic for the independence test in (1):

$$
\hat{T}_{n, p}=\frac{p}{\hat{A}_{p}} \max _{1 \leq i<j \leq n} \frac{T_{i j}^{2}}{\hat{\psi}_{i i} \hat{\psi}_{j j}} .
$$

2.2. Estimation of $A_{p}$ and $\|\boldsymbol{\Sigma}\|_{\mathrm{F}}^{2}$ from correlated samples. The estimation of $\|\boldsymbol{\Sigma}\|_{\mathrm{F}}^{2}$ finds many applications and has been studied in several works [Bai and Saranadasa (1996), Chen and Qin (2010), Fan, Rigollet and Wang (2015)]. However, all these works rely on the sample independence assumption. In particular, Fan, Rigollet and Wang (2015) proved that the simple plug-in procedure based on threshold estimators are minimax optimal over a large class of covariance matrices. Moreover, the threshold level in Fan, Rigollet and Wang (2015) takes the form of $C \sqrt{\frac{\log p}{n}}$, where the constant $C$ needs to be carefully tuned to achieve good performance in practice. A cross-validation (CV) procedure was suggested; however, there is no theoretical justification for such a $\mathrm{CV}$ procedure. In this section, we introduce a threshold estimator for $\|\Sigma\|_{\mathrm{F}}^{2}$ with an explicit threshold level, which is completely data-driven without any tuning and automatically adaptive to the correlation among samples. We will show in Theorem 2.1 that the obtained estimator is ratio-consistent for correlated samples.

Let us define the (column) sample covariance matrix $\hat{\Sigma}=\left(\hat{\sigma}_{i j}\right)_{1 \leq i, j \leq p}$ with $\hat{\sigma}_{i j}=\frac{1}{n-1} \sum_{k=1}^{n}\left(X_{k i}-\bar{X}_{i}\right)\left(X_{k j}-\bar{X}_{j}\right)$ and sample correlation coefficient $\hat{\rho}_{i j}=$ $\hat{\sigma}_{i j} / \sqrt{\hat{\sigma}_{i i} \hat{\sigma}_{j j}}$ for $1 \leq i, j \leq p$. Further, define

$$
B_{n}=\frac{\|\boldsymbol{\Psi}\|_{\mathrm{F}}^{2}}{n}=\frac{1}{n} \sum_{1 \leq i, j \leq n} \psi_{i j}^{2},
$$

which quantifies the average correlation among samples. It can be shown that $\frac{\hat{\rho}_{i j}-\rho_{i j}}{\sqrt{B_{n}}\left(1-\hat{\rho}_{i j}^{2}\right)} \Rightarrow N(0,1)$ (see Proposition 3.1 in Section 3 and note that $\hat{\rho}_{i j} \rightarrow \rho_{i j}$ in 
probability). We propose the following threshold estimator $\hat{\boldsymbol{\Sigma}}_{\mathrm{thr}}=\left(\hat{\sigma}_{i j, \mathrm{thr}}\right)_{1 \leq i, j \leq p}$, where

$$
\begin{aligned}
& \hat{\sigma}_{i j, \mathrm{thr}}=\hat{\sigma}_{i j} I\left\{\frac{\left|\hat{\rho}_{i j}\right|}{1-\hat{\rho}_{i j}^{2}} \geq \delta \sqrt{\frac{\hat{B}_{n} \log p}{n}}\right\} \quad \text { for } i \neq j, \\
& \hat{\sigma}_{i i, \mathrm{thr}}=\hat{\sigma}_{i i} \quad \text { for } 1 \leq i \leq p .
\end{aligned}
$$

Here, $\hat{B}_{n}$ is an estimator of $B_{n}$ and $\delta$ can be any constant larger than $\sqrt{2}$. Let $\hat{\boldsymbol{\Psi}}=\left(\frac{p}{\operatorname{tr}(\hat{\boldsymbol{\Sigma}})} \hat{\psi}_{i j}\right)_{1 \leq i, j \leq n}$. Using the approach from Bai and Saranadasa (1996), we construct

$$
\hat{B}_{n}=\frac{1}{n}\left(\|\hat{\boldsymbol{\Psi}}\|_{\mathrm{F}}^{2}-\frac{1}{p}(\operatorname{tr}(\hat{\boldsymbol{\Psi}}))^{2}\right) .
$$

Given the threshold estimator $\hat{\boldsymbol{\Sigma}}_{\mathrm{thr}}=\left(\hat{\sigma}_{i j, \mathrm{thr}}\right)_{1 \leq i, j \leq p}$ in (12), the $\|\boldsymbol{\Sigma}\|_{\mathrm{F}}^{2}$ is estimated by $\left\|\hat{\boldsymbol{\Sigma}}_{\text {thr }}\right\|_{\mathrm{F}}^{2}$ and $A_{p}$ is estimated by

$$
\hat{A}_{p}=\frac{p\left\|\hat{\boldsymbol{\Sigma}}_{\mathrm{thr}}\right\|_{\mathrm{F}}^{2}}{\left(\operatorname{tr}\left(\hat{\boldsymbol{\Sigma}}_{\mathrm{thr}}\right)\right)^{2}} .
$$

Now we will show that $\left\|\hat{\boldsymbol{\Sigma}}_{\text {thr }}\right\|_{\mathrm{F}}^{2}$ and $\hat{A}_{p}$ are ratio-consistent estimators of $\|\boldsymbol{\Sigma}\|_{\mathrm{F}}$ and $A_{p}$, respectively. We first make the following three assumptions throughout this section. Let $\lambda_{\min }(\Sigma)=\lambda_{1} \leq \lambda_{2} \leq \cdots \leq \lambda_{p}=\lambda_{\max }(\Sigma)$ be the eigenvalues of $\boldsymbol{\Sigma}$ and $\lambda_{\min }(\boldsymbol{\Psi})=v_{1} \leq v_{2} \leq \cdots \leq v_{n}=\lambda_{\max }(\boldsymbol{\Psi})$ be eigenvalues of $\boldsymbol{\Psi}$. We make the following standard assumption on eigenvalues:

(C1) We assume that $c^{-1} \leq \lambda_{\min }(\boldsymbol{\Sigma}) \leq \lambda_{\max }(\boldsymbol{\Sigma}) \leq c$ and $c^{-1} \leq \lambda_{\min }(\boldsymbol{\Psi}) \leq$ $\lambda_{\max }(\Psi) \leq c$ for some constant $c>0$.

The condition (C1) is a typical eigenvalue assumption in high-dimensional covariance estimation literature [see the survey Cai, Ren and Zhou (2016) and references therein]. This assumption is natural for many important classes of covariance matrices, for example, bandable, Toeplitz and sparse covariance matrices. There are cases that the assumption $(\mathrm{C} 1)$ is violated, for example, when the covariance matrix has equal correlation structure [i.e., $\boldsymbol{\Sigma}=\rho \cdot \mathbf{1 1}^{\prime}+(1-\rho) \cdot \mathbf{I}_{p \times p}$ for some $\rho \in(0,1)]$. Our result will not hold for such a setting and please refer to Figure 3 in Section 4.1 for the experimental illustrations.

We also note that this condition can be weakened by replacing the constant $c$ by some $c_{p} \rightarrow \infty$ at a certain rate. However, for the sake of simplicity, we do not intend to seek the optimal rate of $c_{p}$. We only mention that this type of constraint on eigenvalues is needed in our problem. Without this type of constraint, $T_{i j}$ in (7) will no longer be asymptotic normal because the Lindeberg's condition for the central limit theorem (CLT) of independent random variables [see the expression of $\hat{\psi}_{i j}$ in equation (67) in the Supplementary Material, Chen and Liu (2018)] is 
violated. Thus, our result on type I error rate control in Proposition 2.3 will no longer hold.

The second condition is also a standard assumption on the norm of each row of $\boldsymbol{\Psi}$ and $\boldsymbol{\Sigma}$.

(C2) For some $0<\tau<2$, assume that $\sum_{k=1}^{n}\left|\psi_{i k}\right|^{\tau} \leq C$ uniformly over each row $1 \leq i \leq n$ and $\sum_{k=1}^{p}\left|\sigma_{j k}\right|^{\tau} \leq C$ uniformly over each row $1 \leq j \leq p$.

Notably, the upper bounds on eigenvalues of $\boldsymbol{\Sigma}$ and $\boldsymbol{\Psi}$ in (C1) only imply the $\ell_{2}$ boundedness of each row of $\boldsymbol{\Psi}$ and $\boldsymbol{\Sigma}$, that is, $\sum_{k=1}^{n}\left|\psi_{i k}\right|^{2} \leq c^{2}$ and $\sum_{k=1}^{p}\left|\sigma_{j k}\right|^{2} \leq$ $c^{2}$. The condition (C2) is stronger than this implication by noticing that $0<\tau<2$. Moreover, when $0<\tau<1$, this assumption becomes the typical weak sparsity assumption in high-dimensional covariance estimation.

The third assumption is on the relationship between $n$ and $p$.

(C3) We assume that $p>c n$ for some universal constant $c>0$ that does not depend on $p$ and $n$. We further assume that $p=\exp \left(o\left(n^{\gamma}\right)\right)$ with $\gamma=(1-\epsilon) \wedge$ $\left(\frac{2}{\tau}-1\right)$ for some $\epsilon>0$.

The first condition $p=p_{n}>c n$ is quite natural in a high-dimensional setting and the second condition $p=\exp \left(o\left(n^{\gamma}\right)\right)$ allows us to deal with an ultra highdimensional setting.

Under these three assumptions, we provide the following theorem, which establishes the ratio consistency of the estimators $\hat{A}_{p}$ and $\left\|\hat{\boldsymbol{\Sigma}}_{\mathrm{thr}}\right\|_{\mathrm{F}}^{2}$.

THEOREM 2.1. Assume that (C1)-(C3) hold. For any $\delta>\sqrt{2}$, we have $\frac{\hat{A}_{p}}{A_{p}}=$ $1+O_{\mathbb{P}}\left(\left(\sqrt{\frac{\log p}{n}}\right)^{\min (1,2-\tau)}\right)$ and $\frac{\left\|\hat{\boldsymbol{\Sigma}}_{\mathrm{thr}}\right\|_{\mathrm{F}}^{2}}{\|\boldsymbol{\Sigma}\|_{\mathrm{F}}^{2}}=1+O_{\mathbb{P}}\left(\left(\sqrt{\frac{\log p}{n}}\right)^{\min (1,2-\tau)}\right)$.

According to Theorem 2.1, we will simply set $\delta=1.42$ in the estimator $\hat{A}_{p}$ in our experiment. In fact, the experimental results are quite robust with respect to the choice of $\delta$. As long as the $\delta$ is above $\sqrt{2}$ and does not take a too large value, the experimental results will not be affected.

Due to the term $\hat{B}_{n}$ in the thresholding level, our estimator is adaptive to the correlations between the samples. We next show that, even when $\boldsymbol{\Sigma}=\mathbf{I}_{p \times p}$, if we use the thresholding level designed for i.i.d. samples without $\hat{B}_{n}$ as in Fan, Rigollet and Wang (2015), the resultant estimator $\tilde{A}_{p}$ will overestimate $A_{p}$, and hence, reduce the power. In particular, define the thresholding estimator

$$
\hat{\mathbf{\Sigma}}_{1}=\left(\hat{\sigma}_{i j, 1}\right) \quad \text { where } \hat{\sigma}_{i j, 1}=\hat{\sigma}_{i j} I\left\{\left|\hat{\sigma}_{i j}\right| \geq \lambda \sqrt{\frac{\log p}{n}}\right\}, i \neq j,
$$

and $\hat{\sigma}_{i i, 1}=\hat{\sigma}_{i i}$. Fan, Rigollet and Wang (2015) showed that, under the i.i.d. assumption, for a large constant-valued $\lambda$ (not depending on $\boldsymbol{\Psi}$ ), $\sum_{i \neq j} \hat{\sigma}_{i j, 1}^{2}$ attains 
the minimax-optimal rate for estimating $\sum_{i \neq j} \sigma_{i j}^{2}$. Let $\tilde{A}_{p}=\frac{p\left\|\hat{\boldsymbol{\Sigma}}_{1}\right\|_{\mathrm{F}}^{2}}{\left(\operatorname{tr}\left(\hat{\boldsymbol{\Sigma}}_{1}\right)\right)^{2}}$. When the samples are correlated, $\left\|\hat{\boldsymbol{\Sigma}}_{1}\right\|_{\mathrm{F}}$ is no longer a ratio consistent estimator for $\|\boldsymbol{\Sigma}\|_{\mathrm{F}}$, and hence results in a poor estimator for $A_{p}$.

Proposition 2.2. Assume that $\mathbf{\Sigma}=\mathbf{I}_{p \times p}$ and (C1)-(C3) hold. For any $\lambda>$ 0 and $v>0$, there is a class of covariance matrices $\boldsymbol{\Psi}$ with $B_{n} \geq 5 \lambda^{2} / v$ such that $\mathbb{P}\left(\tilde{A}_{p} / A_{p} \geq 1+c p^{1-v} / n\right) \rightarrow 1$ as $(n, p) \rightarrow \infty$.

Proposition 2.2 shows that $\tilde{A}_{p}$ will overestimate $A_{p}$ when $p \gg n$. If $\tilde{A}_{p}$ is used to estimate $A_{p}$, then the resultant testing approach will be less powerful than the test with our estimator $\hat{A}_{p}$. We will further show the impact of $\tilde{A}_{p}$ on the power in the simulation.

2.3. Type I error rate control and optimality of statistical power. The following proposition gives the limiting distribution of $T_{i j}$.

PROPOSITION 2.3. Assume that $p \geq$ cn for some constant $c>0$ (which does not depend on $n$ and $p)$ and $(\mathrm{C} 1)$ holds. Under the null $H_{0}$, for $t \in \mathbb{R}$, we have as $(n, p) \rightarrow \infty$

$$
\mathbb{P}\left(\frac{p}{A_{p}} \max _{1 \leq i<j \leq n} \frac{T_{i j}^{2}}{\hat{\psi}_{i i} \hat{\psi}_{j j}}-4 \log n+\log \log n \leq t\right) \rightarrow \exp \left(-\frac{1}{\sqrt{8 \pi}} \exp \left(-\frac{t}{2}\right)\right) .
$$

In Proposition 2.3, the test statistic $\frac{T_{i j}}{\sqrt{\hat{\psi}_{i i} \hat{\psi}_{j j}}}$ can be viewed as a sample correlation coefficient related with $\psi_{i j}$. We first note that Proposition 2.3 cannot be implied by Theorem 4 in Cai and Jiang (2011). Let us denote the sample correlation coefficient by

$$
\widehat{\rho}_{i j}=\frac{\sum_{k=1}^{n}\left(X_{k i}-\bar{X}_{i}\right)\left(X_{k j}-\bar{X}_{j}\right)}{\sqrt{\sum_{k=1}^{n}\left(X_{k i}-\bar{X}_{i}\right)^{2} \sum_{k=1}^{n}\left(X_{k j}-\bar{X}_{j}\right)^{2}}} .
$$

Cai and Jiang (2011) established the limiting distribution of $\max _{|i-j| \geq \tau}\left|\hat{\rho}_{i j}\right|$ for $\tau \geq 1$. Their result requires that $n$ random vectors $\left(X_{k i}, X_{k j}\right)$ for $1 \leq k \leq n$ in the $\operatorname{sum} \sum_{k=1}^{n}\left(X_{k i}-\bar{X}_{i}\right)\left(X_{k j}-\bar{X}_{j}\right)$ in (15) are i.i.d. On the contrary, our statistic $T_{i j}$ is based on $\sum_{k=1}^{p} X_{i k} X_{j k}$, which is a sum of $p$ potentially correlated random variables, no matter under the null or alternatives.

In addition, it is worthwhile to note that Cai and Jiang (2012) revealed an interesting phase transition phenomenon in the limiting distribution of the largest off-diagonal entry of the sample correlation matrix. There are different regimes for large $p$, in which the limiting distributions are different. In contrast, in our problem, there is no such a phase transition phenomenon and the limiting distribution is unified in the high-dimensional setting when $p \geq c n$. To see this more 
clearly, let us assume that $\mathbf{X}^{(k)}$ for $k=1, \ldots, p$, are independent so that the results in Cai and Jiang (2012) are valid. Now, the quantity $p$ is the sample size and $n$ is the dimension. According to Corollary 2.2 of Cai and Jiang (2012), there is a phase transition phenomenon for the distribution of the statistic in Proposition 2.3 (i.e., $\left.\frac{p}{A_{p}} \max _{1 \leq i<j \leq n} \frac{T_{i j}^{2}}{\hat{\psi}_{i i} \hat{\psi}_{j j}}-4 \log n+\log \log n\right)$ between two regimes $\frac{1}{\sqrt{p}} \log n \rightarrow 0$ and $\frac{1}{\sqrt{p}} \log n \rightarrow \alpha \in(0, \infty)$. In our high-dimensional setting, we have $p \geq c n$, which belongs to the first regime $\frac{1}{\sqrt{p}} \log n \rightarrow 0$. Thus, there is no phase transition phenomenon in the high-dimensional setting.

Using Theorem 2.1, we provide the limiting null distribution of our test statistic $\hat{T}_{n, p}$ in the next theorem.

THEOREM 2.4. Assume that (C1)-(C3) hold. Under the null $H_{0}$, we have

$$
\mathbb{P}\left(\hat{T}_{n, p}-4 \log n+\log \log n \leq t\right) \rightarrow \exp \left(-\frac{1}{\sqrt{8 \pi}} \exp \left(-\frac{t}{2}\right)\right)
$$

for $t \in \mathbb{R}$, as $(n, p) \rightarrow \infty$.

REMARK. In Theorem 2.4, we need the additional assumption $p=$ $\exp \left(o\left(n^{\gamma}\right)\right)$ in $(\mathrm{C} 3)$, which is used to obtain a ratio-consistent estimator of $\|\boldsymbol{\Sigma}\|_{\mathrm{F}}^{2}$ and $A_{p}$. If we consider only the limiting distribution of the test statistic under the null (i.i.d. samples), one may use the method from Chen and Qin (2010) to estimate $\|\boldsymbol{\Sigma}\|_{\mathrm{F}}$. The estimator from Chen and Qin (2010) does not require the condition $p=\exp \left(o\left(n^{\gamma}\right)\right)$. However, in terms of statistical power, as we have shown in our simulations, their estimator will overestimate $\|\boldsymbol{\Sigma}\|_{\mathrm{F}}^{2}$ (see Figure $\mathrm{C}$ in the Supplementary Material [Chen and Liu (2018)]) and reduce the power (see Figure 1 in Section 4.1) especially when the correlation among samples is strong. Our estimator is ratio-consistent for both null and alternative (see Theorem 2.1) under the extra condition $p=\exp \left(o\left(n^{\gamma}\right)\right)$. For the thresholding estimator, such a condition on $p$ is necessary. To see this, if $\log p$ is much larger than $n$, then the thresholding level in (12) is much larger than one. Thus, $\hat{\boldsymbol{\Sigma}}_{\text {thr }} \operatorname{becomes} \operatorname{diag}(\hat{\boldsymbol{\Sigma}})$ and $\left\|\hat{\boldsymbol{\Sigma}}_{\mathrm{thr}}\right\|_{\mathrm{F}}^{2}$ will no longer be consistent. As a future direction, it would be interesting to construct a consistent estimator for $\|\Sigma\|_{\mathrm{F}}^{2}$ and $A_{p}$ under the null and alternative simultaneously without the restriction on $p$.

According to Theorem 2.4, for a given significance level $0<\alpha<1$, we reject the null hypothesis whenever $\hat{T}_{n, p} \geq q_{\alpha}+4 \log n-\log \log n$, where $q_{\alpha}$ is the $1-\alpha$ quantile of the type I extreme value distribution with the cumulative distribution function $(\mathrm{CDF}) \exp \left(-\frac{1}{\sqrt{8 \pi}} \exp \left(-\frac{x}{2}\right)\right)$, that is,

$$
q_{\alpha}=-\log (8 \pi)-2 \log \log (1-\alpha)^{-1} .
$$

Theorem 2.4 shows that the proposed test statistic controls the type I error rate at the nominal level asymptotically. 
We now turn to the power analysis. For a given pair of $1 \leq i<j \leq n$, let us define

$$
d_{i j, \Psi}=\psi_{i j}-\frac{\sum_{k=1, \neq i}^{n} \psi_{i k}}{n}-\frac{\sum_{k=1, \neq j}^{n} \psi_{j k}}{n}-\frac{\sum_{1 \leq i \neq j \leq n} \psi_{i j}}{n^{2}(n-1)}
$$

and

$$
d_{n, \Psi}:=\max _{1 \leq i<j \leq n}\left|d_{i j, \Psi}\right| .
$$

The next theorem shows that for a large class of $\boldsymbol{\Psi}$, the null hypothesis will be rejected by our test with probability tending to one.

THEOREM 2.5. Assume that (C1)-(C3) hold and suppose that for some $\delta>2$ and all large enough $n$ and $p$,

$$
d_{n, \Psi} \geq \delta \sqrt{\frac{A_{p} \log n}{p}} .
$$

We have $\mathbb{P}\left(\hat{T}_{n, p}-4 \log n+\log \log n \geq q_{\alpha}\right) \rightarrow 1$ as $(n, p) \rightarrow \infty$.

We next show that our test statistic is minimax rate optimal for statistical power even when $\boldsymbol{\mu}$ and $\boldsymbol{\Sigma}$ are known. To this end, we introduce a class of covariance matrix for $\boldsymbol{\Psi}-\mathcal{F}(\delta)$ for some $\delta>0$ as follows:

$$
\mathcal{F}(\delta)=\left\{\boldsymbol{\Psi} \succ 0: \psi_{i i}=1,1 \leq i \leq n \text { and } d_{n, \Psi} \geq \delta \sqrt{\frac{\log n}{p}}\right\} .
$$

Let $\mathcal{T}_{\alpha}$ be the set of $\alpha$-level tests with $\boldsymbol{\mu}$ and $\boldsymbol{\Sigma}$ being known, that is, $\mathcal{T}_{\alpha}=\left\{T_{\alpha}\right.$ : $\left.\mathbb{P}\left(T_{\alpha}=1 \mid H_{0}\right) \leq \alpha\right\}$. Here, $T_{\alpha}=1$ means the rejection of $H_{0}$.

THEOREM 2.6. Let $\alpha, \beta>0$ and $\alpha+\beta<1$. Assume that (C3) holds. For any $\delta<2$, we have

$$
\varlimsup_{(n, p) \rightarrow \infty} \sup _{T_{\alpha} \in \mathcal{T}_{\alpha}} \inf _{\boldsymbol{\Psi} \in \mathcal{F}(\delta)} \mathbb{P}\left(T_{\alpha}=1\right) \leq 1-\beta
$$

Theorem 2.6 shows that for any $\alpha$-level test $T_{\alpha}$ and any $\delta<2$, there must exist a covariance matrix $\Psi \in \mathcal{F}(\delta)$ such that the probability of rejecting the null is less than $\alpha+\varepsilon$ asymptotically for any $\varepsilon>0$. Theorems 2.5 and 2.6 together show that the proposed test based on $\hat{T}_{n, p}$ is minimax rate optimal by noting that $1 \leq$ $A_{p} \leq C$ for some constant $C>0$ according to the condition (C1). In other words, the order of the lower bound $\sqrt{\frac{\log n}{p}}$ on $d_{n, \Psi}$ cannot be improved, which establishes the minimax-optimal rate for the test. Moreover, when $\boldsymbol{\Sigma}=\mathbf{I}_{p \times p}$, we have $A_{p}=1$, and hence, our test statistic is also minimax constant optimal. 
We further show that (20) is a rather wide class of $\boldsymbol{\Psi}$ in the sense that if (20) does not hold, it will be safe to assume the independence for some applications. In particular, assume that $\boldsymbol{\Psi}$ is a $s_{n}$ sparse matrix, that is, the number of nonzero elements in each row of $\boldsymbol{\Psi}$ is bounded from above by $s_{n}$. Then by (18),

$$
d_{n, \Psi} \geq\left(1-\frac{3 s_{n}}{n}\right) \max _{1 \leq i<j \leq n}\left|\psi_{i j}\right| .
$$

Thus, a sufficient condition for (20) to hold is $s_{n}=o(n)$ and

$$
\max _{1 \leq i<j \leq n}\left|\psi_{i j}\right| \geq \delta \sqrt{\frac{A_{p} \log n}{p}} \quad \text { for some } \delta>2 .
$$

Theorem 2.5 shows that under (22), the null hypothesis will be rejected with probability tending to 1 . In fact, when (22) does not hold, the samples can be safely treated as independent for some applications. Let us take the multiple testing problem of correlations in (3) as an example. As we discussed in the Introduction, the effect of the correlation among samples is quantified by $B_{n}=\frac{\|\boldsymbol{\Psi}\|_{\mathrm{F}}^{2}}{n}$ and when $B_{n} \rightarrow 1$, the limiting distribution of $\hat{\rho}_{i j}$ in (4) will be the same as the limiting distribution of $\hat{\rho}_{i j}$ estimated from independent samples. Indeed, when (22) does not hold and $p \geq c n^{\gamma}$ for some $\gamma>1$, then we have $B_{n} \rightarrow 1$ (note that $\psi_{i i}=1$ for $1 \leq i \leq n)$. Thus, the correlation among samples is asymptotically negligible. We next give a more general result on the relation between the lower bound of $\max _{1 \leq i<j \leq n}\left|\psi_{i j}\right|, n$ and $p$. Here, we only assume that $n \rightarrow \infty$ and $p$ is a function of $n$ (note that $p$ can be a constant). Let

$$
\mathcal{G}(a)=\left\{\boldsymbol{\Psi} \succ 0: \psi_{i i}=1,1 \leq i \leq n \text { and } \max _{1 \leq i<j \leq n}\left|\psi_{i j}\right| \geq a\right\} .
$$

THEOREM 2.7. Let $\alpha, \beta>0$ and $\alpha+\beta<1$. For any a and $p$ satisfying

$$
\left(1-a^{2}\right)^{-p / 2}=o\left(n^{2}\right) \quad \text { as } n \rightarrow \infty,
$$

we have

$$
\varlimsup_{n \rightarrow \infty} \sup _{T_{\alpha} \in \mathcal{T}_{\alpha}} \inf _{\boldsymbol{\Psi} \in \mathcal{G}(a)} \mathbb{P}\left(T_{\alpha}=1\right) \leq 1-\beta
$$

Theorem 2.7 shows that when the dimension $p$ is fixed, it is impossible to reject $H_{0}$ correctly for all $\boldsymbol{\Psi} \in \mathcal{G}(a)$ with probability greater than $\alpha+\varepsilon$, even when the lower bound $\max _{1 \leq i<j \leq n}\left|\psi_{i j}\right|$ is close to one. It is easy to understand since the role of $n$ and $p$ is interchanged in our setting (we are testing an $n \times n$ covariance matrix $\boldsymbol{\Psi}$ with $p$ row samples). It also indicates that the independence test problem (1) is essentially different from the serial independence test in time series analysis. When $a=c / \sqrt{n}$ for some constant $c>0$, we must require $p \geq c_{1} n \log n$ for some $c_{1}>0$ such that the independence testing problem (1) is solvable over $\mathcal{G}(a)$. Note that Pan, Gao and Yang (2014) requires $0<\lim _{n \rightarrow \infty} \frac{p}{n}<\infty$, which means that their method fails to deal with the setting $a \leq c / \sqrt{n}$. On the other hand, by (22), such a setting of minimum signal $a \leq c / \sqrt{n}$ can be solved by the proposed test. 
3. Multiple testing of correlations with correlated observations. As we mentioned in the Introduction, when the independence hypothesis in (1) is rejected, there is potential risk of using inference methods developed based on independence assumption. To illustrate the effect of sample correlations, we study an important high-dimensional problem - the large-scale multiple testing of correlations when the samples are correlated, that is,

$$
H_{0 i j}: \quad \rho_{i j}=0 \quad \text { versus } \quad H_{1 i j}: \quad \rho_{i j} \neq 0 \quad \text { for } 1 \leq i<j \leq p .
$$

When the samples are i.i.d. and normally distributed, the following classical result from Anderson (2003) (Theorem 4.2.4) establishes the limiting distribution of the sample correlation coefficient $\hat{\rho}_{i j}$ :

$$
\frac{\sqrt{n}\left(\hat{\rho}_{i j}-\rho_{i j}\right)}{1-\rho_{i j}^{2}} \Rightarrow N(0,1) .
$$

However, when the samples are correlated, the limiting distribution of $\hat{\rho}_{i j}$ in (25) does not hold. In fact, we can prove the following proposition.

Proposition 3.1. Assume that the condition (C1) holds. We have

$$
\frac{\sqrt{n}\left(\hat{\rho}_{i j}-\rho_{i j}\right)}{\sqrt{B_{n}}\left(1-\rho_{i j}^{2}\right)} \Rightarrow N(0,1),
$$

where $B_{n}=\frac{\|\Psi\|_{\mathrm{F}}^{2}}{n}$.

The term $B_{n}$ is the same quantity as in (11), which represents the average correlation among $n$ samples. When the sample correlation is strong enough to extent such that $B_{n} \geq 1+c>1$, the multiple testing procedure based on (25) [e.g., Benjamini-Hochberg (BH) procedure, Benjamini and Hochberg (1995)] will lead to many false positives. In fact, even when the correct limiting distribution in (26) is used, the resulting test will lose statistical power. For simplicity, let us consider a single testing problem $H_{0 i j}: \rho_{i j}=0$. To control the type I error rate when the samples are correlated, we need a larger critical value for $\hat{\rho}_{i j}$, which is linear in $\sqrt{B_{n}}$. That is, the rejection region should be $\left\{\hat{\rho}_{i j}: \sqrt{n}\left|\hat{\rho}_{i j}\right| \geq \sqrt{B_{n}} \Phi^{-1}(1-\alpha / 2)\right\}$, where $\Phi(\cdot)$ is the standard normal CDF function. Plugging in a ratio-consistent estimator of $B_{n}$ (e.g., using the method developed in Section 2.2 to estimate $\|\boldsymbol{\Psi}\|_{\mathrm{F}}^{2}$ ), we will obtain a test that controls the type I error rate asymptotically. However, such a test will lose statistical power since the length of the acceptance region grows with the strength of the correlation among samples.

In this section, we propose a multiple testing procedure that asymptotically controls the FDR at the nominal level while maintaining good statistical power. Our method is based on the construction of a "sandwich estimator" of $\rho_{i j}$ by decorrelating the samples. In particular, first assume that $\boldsymbol{\mu}$ and $\boldsymbol{\Psi}$ are known. We 
transform the data $\mathbf{X}$ into $\mathbf{Y}=\left(\boldsymbol{Y}_{1}, \ldots, \boldsymbol{Y}_{n}\right):=\left(\mathbf{X}-\boldsymbol{\mu} \mathbf{1}^{\prime}\right) \boldsymbol{\Psi}^{-1 / 2} \sim N\left(0, \boldsymbol{\Sigma} \otimes \mathbf{I}_{n \times n}\right)$ and columns $\boldsymbol{Y}_{k} \in \mathbb{R}^{p}$ for $1 \leq k \leq n$ are i.i.d. from $N(0, \boldsymbol{\Sigma})$. The corresponding "sample" covariance matrix of $\mathbf{Y}$ is ["sample" is quoted here since $\boldsymbol{\mu}$ and $\boldsymbol{\Psi}$ are unknown, and thus $\left(\tilde{\sigma}_{i j, Y}\right)_{p \times p}$ is not a real sample covariance matrix]

$$
\left(\tilde{\sigma}_{i j, Y}\right)_{p \times p}=\frac{1}{n} \sum_{k=1}^{n} \boldsymbol{Y}_{k} \boldsymbol{Y}_{k}^{\prime}=\frac{1}{n}\left(\mathbf{X}-\boldsymbol{\mu} \mathbf{1}^{\prime}\right) \boldsymbol{\Psi}^{-1}\left(\mathbf{X}-\boldsymbol{\mu} \mathbf{1}^{\prime}\right)^{\prime} .
$$

Let $\tilde{\rho}_{i j, Y}=\frac{\tilde{\sigma}_{i j, Y}}{\sqrt{\tilde{\sigma}_{i i, Y} \tilde{\sigma}_{j j, Y}}}$ be the "sample" correlation coefficient matrix. By (25), we have

$$
\frac{\sqrt{n}\left(\tilde{\rho}_{i j, Y}-\rho_{i j}\right)}{1-\rho_{i j}^{2}} \Rightarrow N(0,1)
$$

which implies that the performance of the test statistic $\tilde{\rho}_{i j, Y}$ is the same as that of $\hat{\rho}_{i j}$ for independent samples. By comparing (28) and (26), the asymptotic variance of the sandwich estimator $\tilde{\rho}_{i j, Y}$ is always smaller than that of the sample correlation coefficient as $B_{n} \geq 1$. Therefore, even when $B_{n}$ is bounded by a constant, the sandwich estimator is more powerful. To obtain an estimate of $\tilde{\rho}_{i j, Y}$, we need to estimate $\boldsymbol{\mu}$ and $\boldsymbol{\Psi}^{-1}:=\left(\gamma_{i j}\right)_{n \times n}$. Let $\hat{\boldsymbol{\mu}}=\left(\bar{X}_{1}, \ldots, \bar{X}_{p}\right)^{\prime}$ be the estimator of $\boldsymbol{\mu}$, where $\bar{X}_{i}=\frac{1}{n} \sum_{k=1}^{n} X_{k i}$ for $1 \leq i \leq p$. For estimating $\boldsymbol{\Psi}^{-1}$, we adopt the CLIME estimator proposed in Cai, Liu and Luo (2011). In particular, following Cai, Liu and Luo (2011), we assume that $\boldsymbol{\Psi}^{-1}$ is a weakly sparse matrix, which belongs to the class

$$
\mathcal{G}=\left\{\boldsymbol{\Psi}^{-1}:\left\|\boldsymbol{\Psi}^{-1}\right\|_{l_{1}} \leq M_{n}, \max _{1 \leq i \leq n}\left|\sum_{j=1}^{n} \psi_{i j}\right| \leq N_{n}, \sum_{j=1}^{n}\left|\gamma_{i j}\right|^{q} \leq s_{n}\right\},
$$

where $0 \leq q<1 / 2,\left\|\Psi^{-1}\right\|_{l_{1}}=\max _{1 \leq j \leq n} \sum_{i=1}^{n}\left|\gamma_{i j}\right|$ and the relationship among $M_{n}, N_{n}$ and $s_{n}$ will be specified in the condition of Theorem 3.2. Let $\hat{\mathbb{R}}_{\Psi}=$ $\left(\hat{\psi}_{i j}\right)_{n \times n}$, where $\hat{\psi}_{i j}$ is defined in (5), and $\hat{\boldsymbol{\Gamma}}^{1}=\left(\hat{\gamma}_{i j}^{1}\right)_{n \times n}$ be any optimal solution of the following optimization problem:

$$
\min _{\boldsymbol{\Gamma} \in \mathbb{R}^{n \times n}}\|\boldsymbol{\Gamma}\|_{1} \quad \text { subject to }\left\|\hat{\mathbb{R}}_{\Psi} \boldsymbol{\Gamma}-\mathbf{I}_{n \times n}\right\|_{\infty} \leq \lambda_{n, p} .
$$

Here, $\lambda_{n, p}=c M_{n}\left(\frac{N_{n}}{n}+\sqrt{\frac{\log n}{p}}\right), c$ is a sufficiently large constant, $\|\boldsymbol{\Gamma}\|_{1}=$ $\sum_{1 \leq i, j \leq n}\left|\gamma_{i j}\right|$ and $\|\mathbf{A}\|_{\infty}=\max _{1 \leq i, j \leq n}\left|a_{i j}\right|$ for matrix $\mathbf{A}=\left(a_{i j}\right)_{n \times n}$. We note that in the estimation of $\Psi^{-1}$, each row of $\mathbf{X}$ is treated as a sample, and thus the sample size is $p$ and the dimensionality is $n$. The estimator of $\boldsymbol{\Psi}^{-1}, \hat{\boldsymbol{\Gamma}}=\left(\hat{\gamma}_{i j}\right)_{n \times n}$, is obtained by a symmetrization of $\hat{\boldsymbol{\Gamma}}^{1}: \hat{\gamma}_{i j}=\hat{\gamma}_{i j}^{1} I\left\{\left|\hat{\gamma}_{i j}^{1}\right| \leq\left|\hat{\gamma}_{j i}^{1}\right|\right\}+\hat{\gamma}_{j i}^{1} I\left\{\left|\hat{\gamma}_{i j}^{1}\right|>\right.$ $\left.\left|\hat{\gamma}_{j i}^{1}\right|\right\}$. Based on the estimated $\hat{\boldsymbol{\mu}}$ and $\hat{\boldsymbol{\Gamma}}$, we define the "sandwich estimator" of 


$$
\begin{array}{r}
\left(\tilde{\sigma}_{i j, Y}\right)_{p \times p},\left(\hat{\sigma}_{i j, Y}\right)_{p \times p}=\frac{1}{n}\left(\mathbf{X}-\hat{\boldsymbol{\mu}} \mathbf{1}^{\prime}\right) \hat{\boldsymbol{\Gamma}}\left(\mathbf{X}-\hat{\boldsymbol{\mu}} \mathbf{1}^{\prime}\right)^{\prime} \text { with each } \\
\hat{\sigma}_{i j, Y}=\frac{1}{n}\left(\mathbf{X}_{\cdot, i}-\bar{X}_{i} \mathbf{1}\right)^{\prime} \hat{\boldsymbol{\Gamma}}\left(\mathbf{X} \cdot, j-\bar{X}_{j} \mathbf{1}\right),
\end{array}
$$

where $\mathbf{X}_{\cdot, i}=\left(X_{1 i}, \ldots, X_{n i}\right)^{\prime}$ is the $i$ th column of $\mathbf{X}$. The corresponding correlation coefficient

$$
\hat{\rho}_{i j, Y}=\frac{\hat{\sigma}_{i j, Y}}{\sqrt{\hat{\sigma}_{i i, Y} \hat{\sigma}_{j j, Y}}} .
$$

We note that the "sandwich estimator" $\hat{\rho}_{i j, Y}$ is related to the Knorm correlation proposed by Teng and Huang (2009), which estimates $\boldsymbol{\Psi}^{-1}$ in $\tilde{\rho}_{i j, Y}$ by the inverse of maximum likelihood estimator (MLE) of $\boldsymbol{\Psi}$. However, there is no closed-form solution for MLE of the matrix-variate normal distribution. So it is difficult to develop limiting distribution results for the Knorm correlation in high-dimensional settings.

In the proof of Theorem 3.2, we will show that $\sqrt{n} \max _{1 \leq i \leq j \leq p}\left|\hat{\rho}_{i j, Y}-\tilde{\rho}_{i j, Y}\right|=$ $o_{\mathbb{P}}(1 / \sqrt{\log p})$. Combining it with (28), we have $\frac{\sqrt{n}\left(\hat{\rho}_{i j, Y}-\rho_{i j}\right)}{1-\rho_{i j, Y}^{2}} \Rightarrow N(0,1)$. Therefore, for each single test problem $H_{0 i j}: \rho_{i j}=0$, we propose the test statistic

$$
\hat{T}_{i j}=\sqrt{n} \hat{\rho}_{i j, Y}
$$

and the null $H_{0 i j}$ is rejected when $\left|\hat{T}_{i j}\right| \geq t$ for some threshold level $t>0$.

To implement the large-scale multiple testing of correlations, we adopt the popular BH method [Benjamini and Hochberg (1995)]. In particular, we need to search for a threshold $\hat{t}$ for $\left|\hat{T}_{i j}\right|$ that controls the false discovery proportion (FDP) and false discovery rate (FDR) defined as follows while rejecting as many hypotheses as possible:

$$
\mathrm{FDP}=\frac{\sum_{(i, j) \in \mathcal{H}_{0}} I\left\{\left|\hat{T}_{i j}\right| \geq \hat{t}\right\}}{\max \left\{\sum_{1 \leq i<j \leq p} I\left\{\left|\hat{T}_{i j}\right| \geq \hat{t}\right\}, 1\right\}} \quad \text { and } \quad \mathrm{FDR}=\mathbb{E}(\mathrm{FDP}),
$$

where $\mathcal{H}_{0}=\left\{(i, j): \rho_{i j}=0,1 \leq i<j \leq p\right\}$ is the set of null. Therefore, an ideal choice of the threshold level for a pre-specified significance level $0<\alpha<1$ should be

$$
\hat{t}_{\text {orc }}=\inf \left\{t>0: \frac{\sum_{(i, j) \in \mathcal{H}_{0}} I\left\{\left|\hat{T}_{i j}\right| \geq t\right\}}{\max \left\{\sum_{1 \leq i<j \leq p} I\left\{\left|\hat{T}_{i j}\right| \geq t\right\}, 1\right\}} \leq \alpha\right\} .
$$

The oracle threshold level $\hat{t}_{\text {orc }}$ cannot be computed since $\mathcal{H}_{0}$ is unknown. Nevertheless, since $\hat{T}_{i j} \Rightarrow N(0,1)$ under the null $\rho_{i j}=0$, the numerator in (33), $\sum_{(i, j) \in \mathcal{H}_{0}} I\left\{\left|\hat{T}_{i j}\right| \geq t\right\}$, can be approximated by $2(1-\Phi(t)) \operatorname{Card}\left(\mathcal{H}_{0}\right)$. The quantity $\operatorname{Card}\left(\mathcal{H}_{0}\right)$ can be further bounded from above by $\left(p^{2}-p\right) / 2$ and such an upper bound is good when $\boldsymbol{\Sigma}$ is sparse, which is a common setup. Therefore, we propose the following threshold level $\hat{t}$ and the corresponding multiple testing procedure. 
Multiple Testing Procedure. For a given $0<\alpha<1$, let

$$
\hat{t}=\inf \left\{t \geq 0: \frac{(1-\Phi(t))\left(p^{2}-p\right)}{\max \left\{\sum_{1 \leq i<j \leq p} I\left\{\left|\hat{T}_{i j}\right| \geq t\right\}, 1\right\}} \leq \alpha\right\} .
$$

For $1 \leq i<j \leq p$, we reject $H_{0 i j}$ if $\left|\hat{T}_{i j}\right| \geq \hat{t}$.

The next theorem shows that the proposed procedure controls the FDP and FDR at level $\alpha$ asymptotically. Recall the definition of $\mathcal{H}_{0}$. Let $h_{0}=\operatorname{Card}\left(\mathcal{H}_{0}\right), \mathcal{H}_{1}=$ $\left\{(i, j): \rho_{i j} \neq 0,1 \leq i<j \leq p\right\}, h_{1}=\operatorname{Card}\left(\mathcal{H}_{1}\right)$ and $h=\left(p^{2}-p\right) / 2$. For a given $\gamma>0$, we further define the following sets:

$$
\mathcal{A}_{i}(\gamma)=\left\{j: 1 \leq j \leq p, j \neq i,\left|\rho_{i j}\right| \geq(\log p)^{-2-\gamma}\right\}, \quad 1 \leq i \leq p .
$$

THEOREM 3.2. Assume that the condition (C1) holds, $p \leq n^{r}$ for some $r>0$, and $\boldsymbol{\Psi}^{-1} \in \mathcal{G}$ defined in (29) with

$$
\begin{aligned}
& \frac{p}{\left(n s_{n}^{2}(\log p)^{3}\right)^{1 /(1-q)} M_{n}^{4} \log n} \rightarrow \infty \text { and } \\
& s_{n}=o\left(\frac{n^{1 / 2-q}}{M_{n}^{2-2 q} N_{n}^{1-q}(\log p)^{3 / 2}}\right) .
\end{aligned}
$$

Suppose that $h_{1} \leq \kappa h$ for some $\kappa<1$,

$$
\operatorname{Card}\left\{(i, j): 1 \leq i<j \leq p,\left|\rho_{i j}\right| \geq 4 \sqrt{\log p / n}\right\} \geq \sqrt{\log \log p},
$$

and $\max _{1 \leq i \leq p} \operatorname{Card}\left(\mathcal{A}_{i}(\gamma)\right)=O\left(p^{\rho}\right)$ for some $\rho<1 / 2$ and $\gamma>0$. We have

$$
\lim _{(n, p) \rightarrow \infty} \frac{\mathrm{FDR}}{\alpha h_{0} / h}=1 \quad \text { and } \quad \frac{\mathrm{FDP}}{\alpha h_{0} / h} \rightarrow 1 \quad \text { in probability as }(n, p) \rightarrow \infty \text {. }
$$

We briefly comment on the condition in Theorem 3.2. We note that in the estimation of $\Psi^{-1}, n$ plays the role of dimensionality and $p$ plays the role of the sample size. The condition in (36) ensures that $p$ is sufficiently large so that the estimation of $\Psi^{-1}$ is accurate. On the other hand, the assumption that $p$ is sufficiently large is also natural for high-dimensional applications (e.g., genetic studies). The assumption that $h_{1} \leq \kappa h$ for some $\kappa<1$ is necessary. Since if $h_{0}=o(h)$, then almost all of $\rho_{i j}$ are nonzeros and simply rejecting all the hypotheses will lead to FDR $\rightarrow 0$. The condition in (37), which is only slightly stronger than the condition that the number of true alternatives goes to infinity, is a nearly necessary condition. In fact, Proposition 2.1 in Liu and Shao (2014) shows that if the number of true alternatives is fixed, then it is impossible for the $\mathrm{BH}$ method to control the FDP with probability tending to one at any desired level. The condition on $\max _{1 \leq i \leq p} \operatorname{Card}\left(\mathcal{A}_{i}(\gamma)\right)$ is essentially a sparsity condition for $\boldsymbol{\Sigma}$. In particular, when $p \geq n^{r_{1}}$ with $r_{1}>1$ and the number of nonzero entries in each row of $\Sigma$ is on the order of $\sqrt{n}$ (which is a common assumption for sparse $\Sigma$ ), then the condition on $\max _{1 \leq i \leq p} \operatorname{Card}\left(\mathcal{A}_{i}(\gamma)\right)$ automatically holds. 
4. Numerical results. In this section, we provide numerical results to demonstrate the performance of the proposed test methods. Due to space constraints, some simulations and real experiments are provided in the Supplementary Material [Chen and Liu (2018)]. Recall that the $p \times n$ data matrix $\mathbf{X}$ follows a matrixvariate normal distribution $N\left(\boldsymbol{\mu} \mathbf{1}^{\prime}, \boldsymbol{\Sigma} \otimes \boldsymbol{\Psi}\right)$. The matrix $\boldsymbol{\Sigma}$ (and $\boldsymbol{\Psi}$ ) is generated from one of the following classes of matrices:

1. Auto-correlation matrix where $\sigma_{i j}=\rho^{|i-j|}$ and $\rho$ is set to $0.2,0.5$ or 0.8 . The larger the parameter $\rho$ is, the stronger the correlation.

2. Banded matrix ("band" for short) where $\sigma_{i i}=1, \sigma_{i, i+1}=\sigma_{i+1, i}=0.6$, $\sigma_{i, i+2}=\sigma_{i+2, i}=0.3$ and $\sigma_{i j}=0$ for $|i-j| \geq 3$.

3. Block diagonal matrix ("block" for short) where the main diagonal blocks are $10 \times 10$ square matrices and off-diagonal blocks are zeros matrices. A $10 \times 10$ main diagonal block $\mathbf{B}=\left(b_{i j}\right)_{10 \times 10}$ has $b_{i i}=1$ and $b_{i j}=0.5$ when $i \neq j$.

In simulations, we fix $\mu=0$ and the level of significance $\alpha=0.05$.

4.1. Independence test. We consider the independence test problem in (1). All the reported empirical sizes and powers are averaged over 5000 independent replications. In Table 1, we consider relatively large $n$ and $p$ and show the empirical type I error rate (a.k.a. the empirical size) of the proposed test statistics $\hat{T}_{n, p}$ in (10) under the null when $\boldsymbol{\Psi}=\mathbf{I}_{n \times n}$. From Table 1, as the sample size $n$ and dimension $p$ increase, the empirical type I error rates get closer to the nominal level of 0.05 , which verifies the validity of the proposed test statistics shown in Theorem 2.4.

TABLE 1

Empirical type I error rates for testing independence based on 5000 replications with $\alpha=0.05$

\begin{tabular}{|c|c|c|c|c|c|c|}
\hline$n$ & $\Sigma$ & $\Psi$ & $p=1000$ & $p=2000$ & $p=5000$ & $p=10,000$ \\
\hline \multirow[t]{5}{*}{200} & $0.2^{|i-j|}$ & $\mathbf{I}_{n \times n}$ & 0.046 & 0.046 & 0.042 & 0.043 \\
\hline & $0.5^{|i-j|}$ & $\mathbf{I}_{n \times n}$ & 0.040 & 0.049 & 0.049 & 0.050 \\
\hline & $0.8^{|i-j|}$ & $\mathbf{I}_{n \times n}$ & 0.045 & 0.048 & 0.055 & 0.058 \\
\hline & band & $\mathbf{I}_{n \times n}$ & 0.031 & 0.032 & 0.035 & 0.043 \\
\hline & block & $\mathbf{I}_{n \times n}$ & 0.014 & 0.025 & 0.030 & 0.035 \\
\hline \multirow[t]{5}{*}{500} & $0.2^{|i-j|}$ & $\mathbf{I}_{n \times n}$ & 0.034 & 0.041 & 0.042 & 0.046 \\
\hline & $0.5^{|i-j|}$ & $\mathbf{I}_{n \times n}$ & 0.037 & 0.046 & 0.041 & 0.049 \\
\hline & $0.8^{|i-j|}$ & $\mathbf{I}_{n \times n}$ & 0.028 & 0.050 & 0.048 & 0.055 \\
\hline & band & $\mathbf{I}_{n \times n}$ & 0.032 & 0.035 & 0.038 & 0.040 \\
\hline & block & $\mathbf{I}_{n \times n}$ & 0.016 & 0.025 & 0.041 & 0.044 \\
\hline \multirow[t]{5}{*}{1000} & $0.2^{|i-j|}$ & $\mathbf{I}_{n \times n}$ & 0.039 & 0.035 & 0.048 & 0.044 \\
\hline & $0.5^{|i-j|}$ & $\mathbf{I}_{n \times n}$ & 0.035 & 0.042 & 0.056 & 0.054 \\
\hline & $0.8^{|i-j|}$ & $\mathbf{I}_{n \times n}$ & 0.026 & 0.040 & 0.051 & 0.050 \\
\hline & band & $\mathbf{I}_{n \times n}$ & 0.029 & 0.037 & 0.040 & 0.045 \\
\hline & block & $\mathbf{I}_{n \times n}$ & 0.016 & 0.024 & 0.035 & 0.041 \\
\hline
\end{tabular}


Recalling in the construction of $\hat{T}_{n, p}$ (in particular, in the term $\hat{A}_{p}$ ), we threshold the sample covariance matrix $\hat{\boldsymbol{\Sigma}}$ as in (12), where the threshold level involves the estimator $\hat{B}_{n}$ of $B_{n}=\|\Psi\|_{\mathrm{F}}^{2} / n$. We compare the empirical sizes of the test statistics in the same form as $\hat{T}_{n, p}$ in (10) but using different estimators (listed as follows) of $\|\boldsymbol{\Sigma}\|_{\mathrm{F}}^{2}$ in estimating $A_{p}=\frac{p\|\boldsymbol{\Sigma}\|_{\mathrm{F}}^{2}}{(\operatorname{tr}(\boldsymbol{\Sigma}))^{2}}$ :

1. CV [Fan, Rigollet and Wang (2015)]: plugin estimator based on thresholded $\boldsymbol{\Sigma}$ with the threshold level tuned by cross validation $(\mathrm{CV})$.

2. Bai: method proposed by Bai and Saranadasa (1996).

3. CQ: method proposed by Chen and Qin (2010).

4. $\hat{B}_{n}$ : plugin estimator based on thresholded $\Sigma$ as in (12) with the proposed estimator $\hat{B}_{n}$ for setting the threshold level.

We would like to make it clear that for the ease of presentation, the acronyms $C V$, Bai and CQ refer to the proposed test statistics in the form of $\hat{T}_{n, p}$ while using the corresponding method to construct the estimator of $\|\Sigma\|_{\mathrm{F}}^{2}$ in $A_{p}$.

In Table 2, we show the comparison results when $n=50$ or 100 and $p=1000$. Of note, we only present smaller $n$ and $p$ cases since the computational cost of CV and CQ are expensive for large $n$ and $p$ and the case $n=50 / 100$ and $p=1000$ has been sufficient to demonstrate the points below. We show that the CV cannot control the type I error below the nominal level 0.05. The CQ leads the type I error rates that are closest to the nominal level. However, as we will show later, it has a lower statistical power. The empirical sizes of the proposed test statistics (with the thresholding level $\hat{B}_{n}$ in estimating $\|\Psi\|_{\mathrm{F}}^{2}$ ) are below the nominal level, which shows that the proposed test statistic is conservative when $n$ and $p$ are small. This results from the slow rate of convergence in distribution for the max-type test statistics [Liu, Lin and Shao (2008)]. For small $n$ and $p$, one useful way to make

TABLE 2

Comparison of empirical type I error rates for testing independence when $p=1000$ and $\alpha=0.05$

\begin{tabular}{ccccccc}
\hline $\boldsymbol{n}$ & $\boldsymbol{\Sigma}$ & $\boldsymbol{\Psi}$ & $\mathbf{C V}$ & Bai & $\mathbf{C Q}$ & $\hat{\boldsymbol{B}}_{\boldsymbol{n}}$ \\
\hline 50 & $0.2^{|i-j|}$ & $\mathbf{I}_{n \times n}$ & 0.029 & 0.001 & 0.038 & 0.002 \\
& $0.5^{|i-j|}$ & $\mathbf{I}_{n \times n}$ & 0.129 & 0.004 & 0.039 & 0.008 \\
& $0.8^{|i-j|}$ & $\mathbf{I}_{n \times n}$ & 0.157 & 0.010 & 0.022 & 0.037 \\
& band & $\mathbf{I}_{n \times n}$ & 0.072 & 0.005 & 0.033 & 0.007 \\
& block & $\mathbf{I}_{n \times n}$ & 0.249 & 0.011 & 0.028 & 0.017 \\
& $0.2^{|i-j|}$ & $\mathbf{I}_{n \times n}$ & 0.070 & 0.017 & 0.041 & 0.028 \\
& $0.5^{|i-j|}$ & $\mathbf{I}_{n \times n}$ & 0.118 & 0.020 & 0.036 & 0.034 \\
& $0.8^{|i-j|}$ & $\mathbf{I}_{n \times n}$ & 0.101 & 0.019 & 0.025 & 0.049 \\
& band & $\mathbf{I}_{n \times n}$ & 0.066 & 0.017 & 0.034 & 0.025 \\
& block & $\mathbf{I}_{n \times n}$ & 0.068 & 0.017 & 0.023 & 0.016 \\
\hline
\end{tabular}


the test less conservative is to adopt the critical value from a Monte-Carlo simulation instead of the one derived from the limiting distribution. In particular, we can generate $M$ (e.g., $M=10,000$ in our simulation) replications of $p \times n$ data matrix, where each one is randomly drawn from $N\left(\mathbf{0}, \mathbf{I}_{p \times p} \otimes \mathbf{I}_{n \times n}\right)$ under the null. We compute the corresponding test statistics $\hat{T}_{n, p}^{(i)}, 1 \leq i \leq M$, for each randomly generated data matrix and let $c_{\alpha}$ be the $(1-\alpha)$-quantile of the empirical distribution $\frac{1}{M} \sum_{i=1}^{M} I\left\{\hat{T}_{n, p}^{(i)} \leq t\right\}$. We reject the null whenever the our test statistic $\hat{T}_{n, p} \geq c_{\alpha}$ (note that the statistic is the same and only the critical value is changed). As shown in the additional experimental results in Section E.1 in the Supplementary Material [Chen and Liu (2018)], using a Monte-Carlo based critical value will push the empirical size closer to the nominal $\alpha$ when $n$ and $p$ are small.

Then we compare statistical power of the proposed test procedure when using different estimators of $\|\boldsymbol{\Sigma}\|_{\mathrm{F}}^{2}$ in our test statistic. In particular, we first consider $\boldsymbol{\Psi}=\left(\rho^{|i-j|}\right)_{n \times n}$ and vary the parameter $\rho$ from 0.55 to 0.85 . The larger the $\rho$ is, the stronger the correlation among samples. For different types of $\boldsymbol{\Sigma}$, the empirical powers are all $100 \%$ for our method (Figure 1). The powers using Bai and CQ drop to zeros when $\rho$ becomes larger than 0.7 . Since both methods for estimating $\|\Sigma\|_{\mathrm{F}}^{2}$ are developed under the i.i.d. assumption, when the sample correlation becomes stronger, the estimation of $\|\Sigma\|_{\mathrm{F}}^{2}$ is inaccurate, which leads to inferior statistical powers. The CV-based thresholding method has maintained statistical power $100 \%$ for a wider range of $\rho$. However, we note that the CV fails to control the type I error rate as shown in Table 2. In Section E.2 in the Supplementary Material [Chen and Liu (2018)], we further demonstrate the superiority of using the proposed estimator for $\|\boldsymbol{\Sigma}\|_{\mathrm{F}}^{2}$ in terms of empirical powers when $\boldsymbol{\Psi}$ is a block diagonal matrix.

It is also of interest to investigate the performance of the proposed test statistics when $n$ and $p$ are comparable. We vary $p$ from 50 to 2000 and consider four settings for the sample size, $n=0.5 p, n=p, n=2 p$ and $n=3 p$. We set $\boldsymbol{\Sigma}=\left(0.5^{|i-j|}\right)_{i, j}$ and show the empirical type I error rates and powers for different $\boldsymbol{\Psi}$ in Figure 2. As one can see from Figure 2(a), the empirical type I error rates are approaching the nominal level $\alpha=0.05$ as $p$ increases. Notably, when the ratio between $n$ and $p$ increases, the test statistic becomes more conservative. From Figure 2(b)-2(d), although the powers are low when $p$ is very small (i.e., $p=50$ ), they are $100 \%$ for moderate and large $p$. This simulation study suggests that the proposed independence test performs reasonably well when $n$ and $p$ are comparable.

Moreover, we consider the setting in which $\boldsymbol{\Sigma}$ does not satisfy the conditions (C1)-(C2). In particular, we choose an equicorrelation covariance matrix $\boldsymbol{\Sigma}=0.85 \cdot \mathbf{1 1}^{\prime}+0.15 \cdot \mathbf{I}_{p \times p}$, which enforces very strong correlation among every pairs of variables. It is easy to see that $\lambda_{\max }(\boldsymbol{\Sigma})=0.85 \cdot p+0.15$ and $\sum_{k=1}^{p}\left|\sigma_{j k}\right|^{\tau}=1+0.85^{\tau} \cdot(p-1)$. Both quantities are linear in $p$, and thus cannot be bounded by constants as $p$ grows, which violates the assumptions (C1)-(C2). Hence, it is expected that the results of type I error rate control in Proposition 2.3 


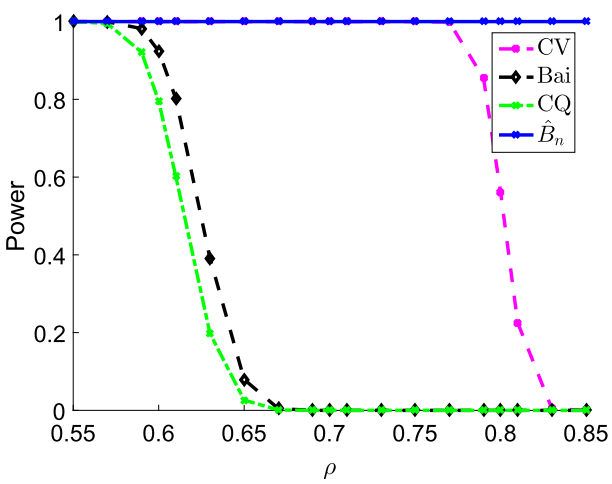

(a) $\boldsymbol{\Sigma}=\left(0.5^{|i-j|}\right)_{i, j}$

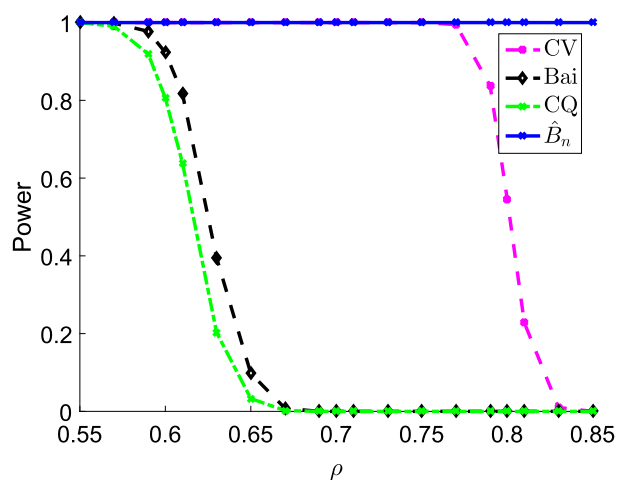

(b) Banded $\boldsymbol{\Sigma}$

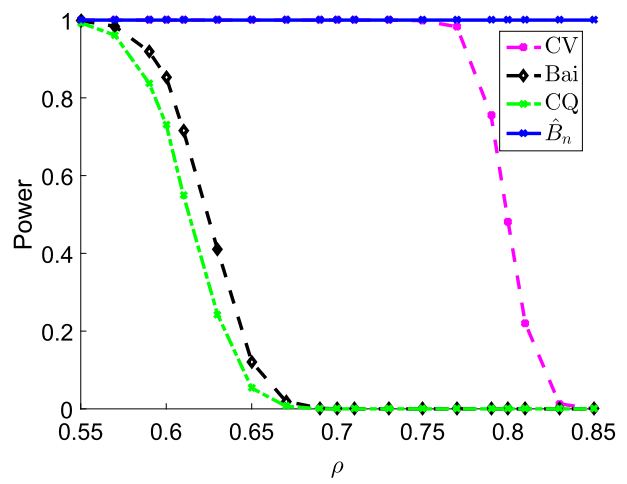

(c) Block $\boldsymbol{\Sigma}$

FIG. 1. Comparison of empirical powers when using different estimators of $\|\boldsymbol{\Sigma}\|_{\mathrm{F}}^{2}$ in $\hat{A}_{p}$. The $\boldsymbol{\Psi}$ matrix is set to the auto-correlation matrix where $\psi_{i j}=\rho^{|i-j|}$ and we vary $\rho$ (corresponding to $x$-axis in each figure) from 0.55 to 0.85 . Here, $n=50, p=1000$ and $\alpha=0.05$.

and Theorem 2.4 will not hold for this model. This is verified by our simulation study. In particular, we vary $n$ and $p$ and show the type I error rates in Figure 3. When $p$ grows and the conditions (C1)-(C2) no longer hold, the type I error rate exceeds the nominal level $\alpha=0.05$ (represented by the green line). Due to space limitations, we relegate the other settings of $\boldsymbol{\Psi}$ and some additional simulation studies for independence testing to Section E in the Supplementary Material [Chen and Liu (2018)], which includes:

1. We compare empirical powers when the $\boldsymbol{\Psi}$ is a block diagonal matrix and demonstrate the superiority of the proposed method.

2. To empirically verify the result in Theorem 2.5 , we consider the case of extremely sparse $\boldsymbol{\Psi}$ where $\psi_{12}=\psi_{21}=\kappa \sqrt{\frac{\log n}{p}}$ and all the other off-diagonal elements are zeros. The experimental results show that for different types of $\boldsymbol{\Sigma}$, the empirical powers all become $100 \%$ as $\kappa$ increases, which demonstrates that 


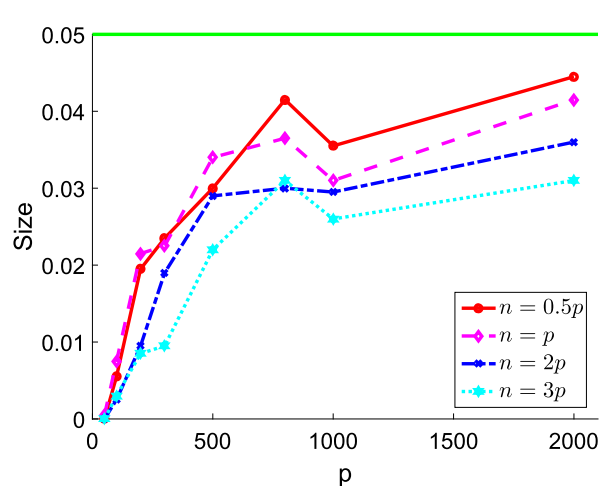

(a) $\boldsymbol{\Psi}=\mathbf{I}_{n \times n}$

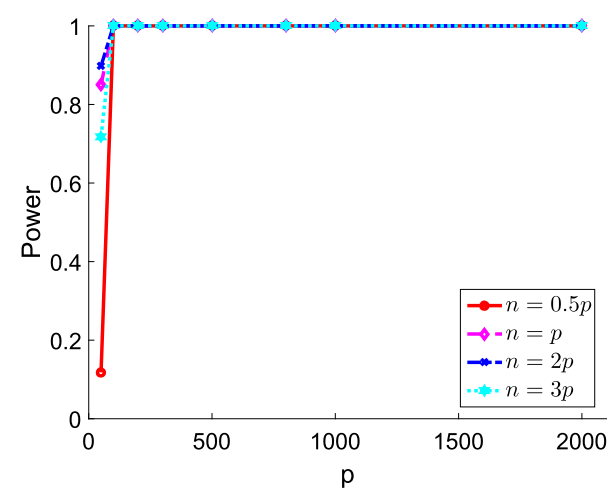

(c) Banded $\Psi$

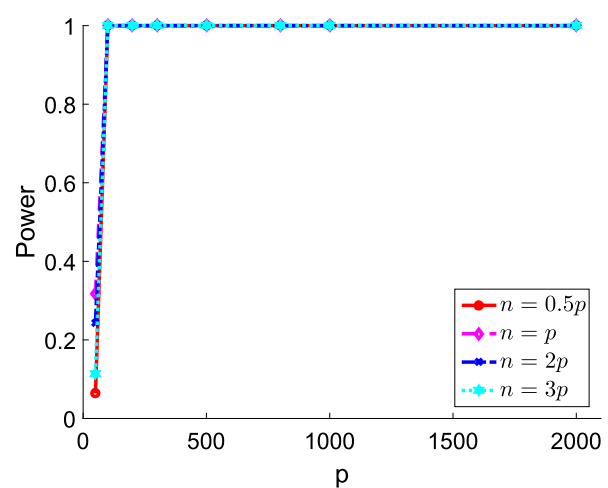

(b) $\boldsymbol{\Psi}=\left(0.5^{|i-j|}\right)_{i, j}$

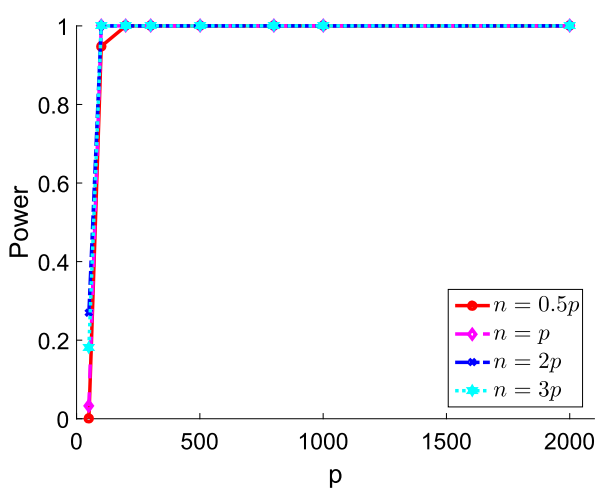

(d) Block $\Psi$

FIG. 2. The empirical type I error rates and powers when $n=0.5 p, n=p, n=2 p$ and $n=3 p$ and $p$ varies from 50 to 2000. Panel (a) shows the empirical type I error rate and the green line indicates the nominal level $\alpha=0.05$. Panels (b)-(d) show the empirical powers for different $\Psi$.

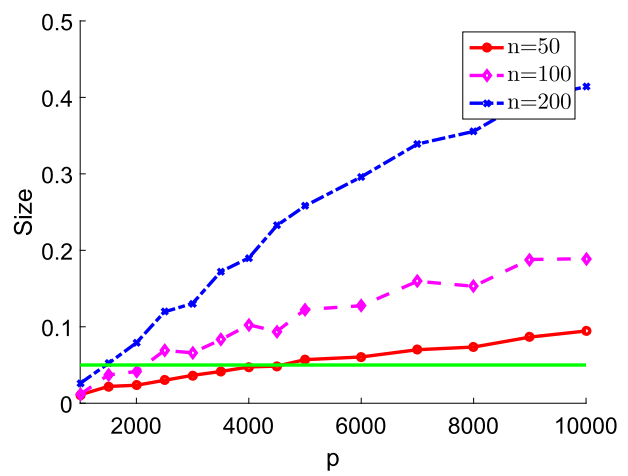

FIG. 3. The empirical type I error rates when $\mathbf{\Sigma}=0.85 \cdot \mathbf{1 1}^{\prime}+0.15 \cdot \mathbf{I}_{p \times p}$ for $n=50,100,200$ and $p$ varying from 1000 to 10,000 . The green line indicates the nominal level $\alpha=0.05$. 
our test statistic can successfully reject the null even when the $\boldsymbol{\Psi}$ is extremely sparse.

3. To provide more intuitive comparisons between different methods for estimating $\|\boldsymbol{\Sigma}\|_{\mathrm{F}}^{2}$, we directly show the relative estimation error under different settings. This experiment demonstrates that the proposed thresholding estimator greatly outperforms its competitors when the correlation among samples is large.

4.2. Large-scale multiple testing of correlations. In this section, we conduct both simulated and real data analysis to demonstrate performance of the proposed "sandwich" estimator in (32) for large-scale multiple testing of correlations in (24).

4.2.1. FDP and power of simulated results. In simulated study, we compare the $\mathrm{BH}$ procedure based on four different estimators of $\rho_{i j}$ :

1. The proposed sandwich estimator $\hat{T}_{i j}\left(\lambda_{n, p}\right)=\sqrt{n} \hat{\rho}_{i j, Y}$ in (32), where $\boldsymbol{\Psi}^{-1}$ is estimated by CLIME [Cai, Liu and Luo (2011)]. Further, we adopt the datadriven approach in Liu (2013) to tune the $\lambda_{n, p}$ in CLIME [see (30)]. In particular, the parameter $\lambda_{n, p}$ is selected by

$$
\hat{\lambda}_{n, p}=\underset{\lambda}{\arg \min } \sum_{k=3}^{9}\left(\frac{\sum_{1 \leq i \neq j \leq p} I\left\{\left|\hat{T}_{i, j}(\lambda)\right| \geq \Phi^{-1}(1-k / 20)\right\}}{k\left(p^{2}-p\right) / 10}-1\right)^{2} .
$$

2. The classical sample correlation estimator $\sqrt{n} \hat{\rho}_{i j}$ based on sample independence assumption.

3. The variance corrected sample correlation estimator $\frac{\sqrt{n} \hat{\rho}_{i j}}{\sqrt{B_{n}}}$, where true $B_{n}=$ $\|\Psi\|_{\mathrm{F}}^{2} / n$ is assumed to be known.

4. The proposed sandwich estimator in (32) with the true $\Psi^{-1}$, which serves as an oracle benchmark.

In Table 3, we report the averaged FDP and power over 100 replications. The matrix $\Sigma$ is chosen to be either banded or block diagonal matrix, both of which are sparse. As we can see from Table 3, the FDPs of the BH procedure based on sandwich estimator are below $\alpha=0.05$. The empirical powers get close to one as the sample size $n$ increases and are only slightly worse than the powers of the oracle benchmark with true $\Psi^{-1}$. For the classical sample correlation estimator $\sqrt{n} \hat{\rho}_{i j}$, the FDP can be very large (e.g., around $50 \%$ when $\psi_{i j}=0.5^{|i-j|}$ and more than $95 \%$ when $\left.\psi_{i j}=0.8^{|i-j|}\right)$. This verifies our result showing that naïvely using the sample correlation estimator developed under the sample independence assumption will lead to many false positives. Using the variance corrected sample correlation estimator $\frac{\sqrt{n} \hat{\rho}_{i j}}{\sqrt{B_{n}}}$ will help reduce the number of false positives and control FDP as shown in Table 3, 
TABLE 3

Averaged FDP and power for testing correlations over 100 replications. Here, $\alpha=0.05$ and $p=1000$

\begin{tabular}{|c|c|c|c|c|c|c|c|c|c|c|}
\hline \multirow[t]{2}{*}{$n$} & \multirow[t]{2}{*}{$\Sigma$} & \multirow[t]{2}{*}{$\Psi$} & \multicolumn{2}{|c|}{$\sqrt{n} \hat{\rho}_{i j, Y}$} & \multicolumn{2}{|c|}{$\sqrt{n} \hat{\rho}_{i j}$} & \multicolumn{2}{|c|}{$\frac{\sqrt{n} \hat{\rho}_{i j}}{\sqrt{\boldsymbol{B}_{n}}}$} & \multicolumn{2}{|c|}{ True $\Psi^{-1}$} \\
\hline & & & FDP & Power & FDP & Power & FDP & Power & FDP & Power \\
\hline \multirow[t]{6}{*}{50} & band & $0.2^{|i-j|}$ & 0.010 & 0.311 & 0.027 & 0.339 & 0.010 & 0.276 & 0.015 & 0.339 \\
\hline & band & $0.5^{|i-j|}$ & 0.009 & 0.308 & 0.403 & 0.378 & 0.000 & 0.000 & 0.015 & 0.340 \\
\hline & band & $0.8^{|i-j|}$ & 0.009 & 0.292 & 0.986 & 0.648 & 0.000 & 0.000 & 0.014 & 0.338 \\
\hline & block & $0.2^{|i-j|}$ & 0.011 & 0.262 & 0.036 & 0.416 & 0.014 & 0.295 & 0.021 & 0.407 \\
\hline & block & $0.5^{|i-j|}$ & 0.011 & 0.257 & 0.366 & 0.504 & 0.000 & 0.000 & 0.021 & 0.410 \\
\hline & block & $0.8^{|i-j|}$ & 0.012 & 0.168 & 0.965 & 0.739 & 0.010 & 0.000 & 0.021 & 0.408 \\
\hline \multirow[t]{6}{*}{100} & band & $0.2^{|i-j|}$ & 0.025 & 0.576 & 0.057 & 0.593 & 0.029 & 0.568 & 0.033 & 0.587 \\
\hline & band & $0.5^{|i-j|}$ & 0.025 & 0.575 & 0.581 & 0.650 & 0.011 & 0.448 & 0.032 & 0.587 \\
\hline & band & $0.8^{|i-j|}$ & 0.025 & 0.556 & 0.986 & 0.795 & 0.000 & 0.000 & 0.032 & 0.587 \\
\hline & block & $0.2^{|i-j|}$ & 0.028 & 0.942 & 0.061 & 0.961 & 0.035 & 0.945 & 0.038 & 0.966 \\
\hline & block & $0.5^{|i-j|}$ & 0.028 & 0.935 & 0.454 & 0.942 & 0.017 & 0.646 & 0.038 & 0.966 \\
\hline & block & $0.8^{|i-j|}$ & 0.030 & 0.820 & 0.963 & 0.924 & 0.000 & 0.000 & 0.038 & 0.966 \\
\hline \multirow[t]{6}{*}{200} & band & $0.2^{|i-j|}$ & 0.036 & 0.839 & 0.072 & 0.852 & 0.039 & 0.820 & 0.041 & 0.854 \\
\hline & band & $0.5^{|i-j|}$ & 0.036 & 0.835 & 0.620 & 0.867 & 0.028 & 0.640 & 0.041 & 0.853 \\
\hline & band & $0.8^{|i-j|}$ & 0.041 & 0.749 & 0.984 & 0.906 & 0.002 & 0.240 & 0.042 & 0.852 \\
\hline & block & $0.2^{|i-j|}$ & 0.034 & 1.000 & 0.071 & 1.000 & 0.041 & 1.000 & 0.043 & 1.000 \\
\hline & block & $0.5^{|i-j|}$ & 0.034 & 1.000 & 0.498 & 1.000 & 0.033 & 0.992 & 0.044 & 1.000 \\
\hline & block & $0.8^{|i-j|}$ & 0.040 & 0.969 & 0.962 & 0.994 & 0.004 & 0.233 & 0.044 & 1.000 \\
\hline
\end{tabular}

which is consistent with our result in Proposition 3.1. However, as we observe from Table 3, even when the true $B_{n}$ is used, the powers of $\frac{\sqrt{n} \hat{\rho}_{i j}}{\sqrt{B_{n}}}$ are quite low, especially when the correlation among samples becomes stronger. The reason for this low power is explained in the paragraph below Proposition 3.1 .

In Table 4, we consider the setting when the samples are i.i.d., in which case the classical sample correlation estimator should be used as it is based on sample independence assumption. We also note that when samples are i.i.d., both the variance corrected sample correlation estimator $\frac{\sqrt{n} \hat{\rho}_{i j}}{\sqrt{B_{n}}}\left(B_{n}=1\right)$ and the sandwich estimator with true $\boldsymbol{\Psi}^{-1}=\mathbf{I}_{n \times n}$ reduce to the classical sample correlation estimator. The power when using the sandwich estimator with the estimated $\Psi^{-1}$ by CLIME is quite close to the power when using the benchmark sample correlation estimator (Table 4), which demonstrates the robustness of the proposed method. 
TABLE 4

Averaged FDP and power for testing correlations over 100 replications when samples are i.i.d. Here, $\alpha=0.05$ and $p=1000$

\begin{tabular}{|c|c|c|c|c|c|c|}
\hline \multirow[t]{2}{*}{$n$} & \multirow[t]{2}{*}{$\Sigma$} & \multirow[t]{2}{*}{$\Psi$} & \multicolumn{2}{|c|}{$\sqrt{n} \hat{\rho}_{i j, Y}$} & \multicolumn{2}{|c|}{$\sqrt{n} \hat{\rho}_{i j}$} \\
\hline & & & FDP & Power & FDP & Power \\
\hline \multirow[t]{2}{*}{50} & band & $\mathbf{I}_{n \times n}$ & 0.009 & 0.318 & 0.014 & 0.341 \\
\hline & block & $\mathbf{I}_{n \times n}$ & 0.012 & 0.293 & 0.021 & 0.408 \\
\hline \multirow[t]{2}{*}{100} & band & $\mathbf{I}_{n \times n}$ & 0.025 & 0.578 & 0.032 & 0.587 \\
\hline & block & $\mathbf{I}_{n \times n}$ & 0.028 & 0.952 & 0.037 & 0.965 \\
\hline \multirow[t]{2}{*}{200} & band & $\mathbf{I}_{n \times n}$ & 0.035 & 0.844 & 0.041 & 0.852 \\
\hline & block & $\mathbf{I}_{n \times n}$ & 0.035 & 1.000 & 0.043 & 1.000 \\
\hline
\end{tabular}

We also conducted real experiments on correlation tests for yeast genomics data and stock data, which are detailed in Section E.5 in the Supplementary Material [Chen and Liu (2018)].

5. Discussion. This paper studies the sample/column independence test and multiple testing of Pearson's correlation coefficients in a high-dimensional setting. The main difficulty in column independence test arises from the correlation among different variables, which is characterized by the covariance matrix $\boldsymbol{\Sigma}$. If $\boldsymbol{\Sigma}$ is known, the data matrix can be transformed as $\boldsymbol{\Sigma}^{-1 / 2} \mathbf{X} \sim N\left(\boldsymbol{\Sigma}^{-1 / 2} \boldsymbol{\mu} \mathbf{1}^{\prime}, \mathbf{I}_{p \times p} \otimes \boldsymbol{\Psi}\right)$, based on which the independence test can be directly carried out using existing approaches [e.g., Jiang (2004), Liu, Lin and Shao (2008)]. However, the covariance matrix $\boldsymbol{\Sigma}$ is unknown. Although the problem of estimating $\boldsymbol{\Sigma}^{-1}$ has been well studied, the optimal convergence rate in matrix $\ell_{1}$-norm is known to be $O\left(s_{p}\left\|\boldsymbol{\Sigma}^{-1}\right\|_{l_{1}} \sqrt{(\log p) / n}\right)$, where $s_{p}$ is the row sparsity level of $\boldsymbol{\Sigma}^{-1}$ [see, e.g., Cai, Liu and Zhou (2016)]. However, such a rate is not fast enough for establishing a limiting null distribution of the test statistic based on the estimated $\boldsymbol{\Sigma}^{-1}$. In particular, from the proof of Theorem 2.4, when using max-type test statistics, to eliminate the effect of the estimation error from $\boldsymbol{\Sigma}^{-1}$ and establish a limiting null distribution, the convergence rate needs to be $o_{\mathbb{P}}(1 / \sqrt{p \log n})$. As $p$ can be $\exp \left(o\left(n^{\gamma}\right)\right)$ for some $\gamma>0$ in an ultra high-dimensional setting, one cannot solve the independence test problem in (1) by simply plugging in the estimator of $\boldsymbol{\Sigma}^{-1}$. On the other hand, when using the row sample correlation matrix $\left(\hat{\psi}_{i j}\right)$ by treating each row of $\boldsymbol{X}$ as a sample, we only need to estimate $\|\boldsymbol{\Sigma}\|_{\mathrm{F}}^{2}$ instead of $\boldsymbol{\Sigma}^{-1}$. The problem of estimating $\|\boldsymbol{\Sigma}\|_{\mathrm{F}}^{2}$ from correlated samples has been successfully addressed in Section 2.2. We would also like to note that in the multiple testing problem of Pearson's correlation coefficients, such a difficulty no longer exists. In fact, when estimating $\boldsymbol{\Psi}^{-1}$ from row samples of $\boldsymbol{X}$, the roles of $n$ and $p$ has interchanged (i.e., the sample size becomes $p$ and the dimensionality becomes $n$ ), 
and thus, the estimation problem is conducted in a relatively lower dimensional setting.

\section{SUPPLEMENTARY MATERIAL}

\section{Supplement to "Testing independence with high-dimensional correlated} samples" (DOI: 10.1214/17-AOS1571SUPP; .pdf). We provide the proofs of all the theorectial results as well as additional simulated and real experimental results.

\section{REFERENCES}

Allen, G. I. and Tibshirani, R. (2012). Inference with transposable data: Modelling the effects of row and column correlations. J. R. Stat. Soc. Ser. B. Stat. Methodol. 74 721-743. MR2965957

Anderson, T. W. (2003). An Introduction to Multivariate Statistical Analysis, 3rd ed. WileyInterscience, Hoboken, NJ. MR1990662

BAI, Z. and SARANADASA, H. (1996). Effect of high dimension: By an example of a two sample problem. Statist. Sinica 6 311-329. MR1399305

BAI, Z., JiAng, D., YAO, J.-F. and ZHENG, S. (2009). Corrections to LRT on large-dimensional covariance matrix by RMT. Ann. Statist. 37 3822-3840. MR2572444

Benjamini, Y. and Hochberg, Y. (1995). Controlling the false discovery rate: A practical and powerful approach to multiple testing. J. R. Stat. Soc. Ser. B. Stat. Methodol. 57 289-300. MR1325392

BIEn, J. and TIBSHIRANi, R. J. (2011). Sparse estimation of a covariance matrix. Biometrika 98 807-820. MR2860325

CAI, T. T. and JIANG, T. (2011). Limiting laws of coherence of random matrices with applications to testing covariance structure and construction of compressed sensing matrices. Ann. Statist. 39 1496-1525. MR2850210

CAI, T. T. and JiAng, T. (2012). Phase transition in limiting distributions of coherence of highdimensional random matrices. J. Multivariate Anal. 107 24-39. MR2890430

CAI, T. and LIU, W. (2011). Adaptive thresholding for sparse covariance matrix estimation. J. Amer. Statist. Assoc. 106 672-684. MR2847949

CAI, T. T. and LIU, W. (2016). Large-scale multiple testing of correlations. J. Amer. Statist. Assoc. 111 229-240. MR3494655

CAI, T., LIU, W. and LUO, X. (2011). A constrained $\ell_{1}$ minimization approach to sparse precision matrix estimation. J. Amer. Statist. Assoc. 106 594-607. MR2847973

CAI, T., LIU, W. and ZHOU, H. (2016). Estimating sparse precision matrix: Optimal rates of convergence and adaptive estimation. Ann. Statist. 44 455-488. MR3476606

CAI, T. T., REN, Z. and ZHOU, H. H. (2016). Estimating structured high-dimensional covariance and precision matrices: Optimal rates and adaptive estimation. Electron. J. Stat. 10 1-59. MR3466172

Carter, S. L., Brechbühler, C. M., Griffin, M. and Bond, A. T. (2004). Gene co-expression network topology provides a framework for molecular characterization of cellular state. Bioinformatics 20 2242-2250.

CHEN, X. and LIU, W. (2018). Supplement to "Testing independence with high-dimensional correlated samples." DOI:10.1214/17-AOS1571SUPP.

CHEN, S. X. and QIN, Y.-L. (2010). A two-sample test for high-dimensional data with applications to gene-set testing. Ann. Statist. 38 808-835. MR2604697

DAWID, A. P. (1977). Spherical matrix distributions and a multivariate model. J. R. Stat. Soc. Ser. B. Stat. Methodol. 39 254-261. MR0518916 
DAWID, A. P. (1981). Some matrix-variate distribution theory: Notational considerations and a Bayesian application. Biometrika 68 265-274. MR0614963

EFron, B. (2009). Are a set of microarrays independent of each other? Ann. Appl. Stat. 3 922-942. MR2750220

FAN, J., Rigollet, P. and WANG, W. (2015). Estimation of functionals of sparse covariance matrices. Ann. Statist. 43 2706-2737. MR3405609

FAng, K. T. and ZhAng, Y. T. (1990). Generalized Multivariate Analysis. Springer, Berlin. MR1079542

HAN, F., CHEN, S. Z. and LIU, H. (2017). Distribution-free tests of independence with applications to testing more structures. Biometrika 104 813-828.

Hirai, M. Y., Sugiyama, K., Sawada, Y., Tohge, T., Obayashi, T., Suzuki, A., Araki, R., Sakurai, N., Suzuki, H., Aoki, K., Goda, H., Nishizawa, O. I., Shibata, D. and SAITO, K. (2007). Omics-based identification of Arabidopsis Myb transcription factors regulating aliphatic glucosinolate biosynthesis. Proc. Natl. Acad. Sci. USA 104 6478-6483.

HoNG, Y. (1998). Testing for pairwise serial independence via the empirical distribution function. J. R. Stat. Soc. Ser. B. Stat. Methodol. 60 429-453. MR1616065

JIANG, T. (2004). The asymptotic distributions of the largest entries of sample correlation matrices. Ann. Appl. Probab. 14 865-880. MR2052906

JIANG, T. and YANG, F. (2013). Central limit theorems for classical likelihood ratio tests for highdimensional normal distributions. Ann. Statist. 41 2029-2074. MR3127857

Johnstone, I. M. (2001). On the distribution of the largest eigenvalue in principal components analysis. Ann. Statist. 29 295-327. MR1863961

Kim, K., Jiang, K., Teng, S. L., Feldman, L. J. and Huang, H. (2012). Using biologically interrelated experiments to identify pathway genes in Arabidopsis. Bioinformatics 28 815-822.

LAM, C. and FAN, J. (2009). Sparsistency and rates of convergence in large covariance matrix estimation. Ann. Statist. 37 4254-4278. MR2572459

LazZeroni, L. and Owen, A. (2002). Plaid models for gene expression data. Statist. Sinica 12 61-86. MR1894189

Ledoit, O. and Wolf, M. (2002). Some hypothesis tests for the covariance matrix when the dimension is large compared to the sample size. Ann. Statist. 30 1081-1102. MR1926169

LeE, H. K., Hsu, A. K., SAJdak, J., QIn, J. and PAvlidis, P. (2004). Coexpression analysis of human genes across many microarray data sets. Genome Res. 14 1085-1094.

LIU, W. (2013). Gaussian graphical model estimation with false discovery rate control. Ann. Statist. 41 2948-2978. MR3161453

LIU, W.-D., LIN, Z. and SHAO, Q.-M. (2008). The asymptotic distribution and Berry-Esseen bound of a new test for independence in high dimension with an application to stochastic optimization. Ann. Appl. Probab. 18 2337-2366. MR2474539

LIU, W. and SHAO, Q.-M. (2014). Phase transition and regularized bootstrap in large-scale $t$-tests with false discovery rate control. Ann. Statist. 42 2003-2025. MR3262475

MURAlidHARAN, O. (2010). Detecting column dependence when rows are correlated and estimating the strength of the row correlation. Electron. J. Stat. 4 1527-1546. MR2747132

NAGAO, H. (1973). On some test criteria for covariance matrix. Ann. Statist. 1 700-709. MR0339405

PAN, G., GAO, J. and YANG, Y. (2014). Testing independence among a large number of highdimensional random vectors. J. Amer. Statist. Assoc. 109 600-612. MR3223736

Rothman, A. J., LevinA, E. and ZHU, J. (2009). Generalized thresholding of large covariance matrices. J. Amer. Statist. Assoc. 104 177-186. MR2504372

Schотт, J. R. (2005). Testing for complete independence in high dimensions. Biometrika $92951-$ 956. MR2234197

Shaw, P., Greenstein, D., Lerch, J., Clasen, L., Lenroot, R., Gogtay, N., Evans, A., RAPOPORT, J. and GIEDD, J. (2006). Intellectual ability and cortical development in children and adolescents. Nature 440 676-679. 
Teng, S. L. and HuAng, H. (2009). A statistical framework to inter functional gene relationships from biologically interrelated microarray experiments. J. Amer. Statist. Assoc. 104 465-473. MR2751431

YIN, J. and LI, H. (2012). Model selection and estimation in the matrix normal graphical model. J. Multivariate Anal. 107 119-140. MR2890437

ZHoU, S. (2014). Gemini: Graph estimation with matrix variate normal instances. Ann. Statist. 42 532-562. MR3210978

Zhu, D., Hero, A. O., QIN, Z. S. and Swaroop, A. (2005). High throughput screening of coexpressed gene pairs with controlled false discovery rate (FDR) and minimum acceptable strength (MAS). J. Comput. Biol. 12 1029-1045.

STERN SCHOOL OF BUSINESS

NEW YORK UNIVERSITY

44 WEST 4TH STREET

NEW York, NEW YorK 10012

USA

E-MAIL: xchen3@stern.nyu.edu
DEPARTMENT OF MATHEMATICS

INSTITUTE OF NATURAL SCIENCES AND MOE-LSC SHANGHAI JIAO TONG UNIVERSITY

SHANGHAI

CHINA

E-MAIL: weidongl@sjtu.edu.cn 